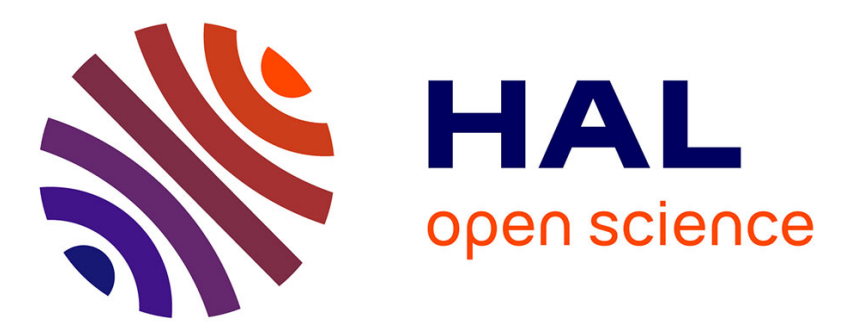

\title{
Subduction of non-Newtonian plates: thin-sheet dynamics of slab necking and break-off
}

\author{
Neil M. Ribe, Bingrui Xu
}

\section{To cite this version:}

Neil M. Ribe, Bingrui Xu. Subduction of non-Newtonian plates: thin-sheet dynamics of slab necking and break-off. Geophysical Journal International, 2020, 220, pp.910-927. 10.1093/gji/ggz500 . hal02903182

\section{HAL Id: hal-02903182 \\ https://hal.science/hal-02903182}

Submitted on $20 \mathrm{Jul} 2020$

HAL is a multi-disciplinary open access archive for the deposit and dissemination of scientific research documents, whether they are published or not. The documents may come from teaching and research institutions in France or abroad, or from public or private research centers.
L'archive ouverte pluridisciplinaire HAL, est destinée au dépôt et à la diffusion de documents scientifiques de niveau recherche, publiés ou non, émanant des établissements d'enseignement et de recherche français ou étrangers, des laboratoires publics ou privés. 


\title{
Subduction of non-Newtonian plates: thin-sheet dynamics of slab necking and break-off
}

\author{
Neil M. Ribe ${ }^{\oplus 1}$ and Bingrui $\mathrm{Xu}^{2}$ \\ ${ }^{1}$ Lab FAST, CNRS, Univ. Paris-Saclay, Bat 530, Campus Univ, F-91400 Orsay, France. E-mail: ribe@fast.u-psud.fr \\ ${ }^{2}$ Department of Aeronautics and Astronautics, University of Fudan, 220 Handan Road, Shanghai 200433, China
}

Accepted 2019 November 4. Received 2019 October 30; in original form 2019 August 1

\begin{abstract}
S UMMAR Y
We use a hybrid boundary-integral/thin-sheet ('BITS') model to investigate the subduction of 2 -D viscous sheets with composite diffusion creep/dislocation creep rheology, with a focus on the conditions required for slab necking and break-off. To validate the model, we show that its predictions of the sinking speed of the slab follow a universal scaling law identical to one previously derived for purely Newtonian sheets. We obtain analytical expressions for the fibre stress resultant and bending moment of the sheet during deformation by pure stretching and pure bending, respectively, and show that the non-Newtonian dislocation creep component of the rheology significantly weakens the sheet for both types of deformation. We solve the BITS equations for two distinct situations: 'free' subduction, in which the slab pulls an attached negatively buoyant plate without hindrance; and 'arrested' subduction that slows or stops when a positively buoyant (continental) portion of the attached plate arrives at the trench. Strong lithospheric thinning is difficult to obtain in free subduction and requires dominantly nonNewtonian rheology, that is large $(>5)$ values of the characteristic ratio $\lambda$ of the Newtonian to the non-Newtonian viscosity. However, during arrested subduction strong thinning leading to break-off occurs for much lower values of $\lambda$, and the point of maximum thinning is at shallower depths. These results are explained by a 1-D viscous dripping model ('DRIP') of a vertical slab with a composite rheology and an arbitrary kinematically prescribed time-dependent convergence rate $U_{0}(t)$. By injecting into DRIP convergence histories predicted by BITS, we find that DRIP reproduces closely the results of the more complicated BITS model. The DRIP model shows that the convergence rate controls slab break-off in two distinct ways. On the one hand, the rate of lithospheric thinning is proportional to the accumulated convergence, that is, the time integral of $U_{0}(t)$. The break-off depth, on the other hand, is controlled by the convergence rate history, being shallower when $U_{0}$ rapidly decreases (arrested subduction) after an initial period of oceanic subduction.
\end{abstract}

Key words: Mantle processes; Numerical modelling; Dynamics of lithosphere and mantle; Subduction zone processes.

\section{INTRODUCTION}

Slab break-off is the process by which the lower portion of a subducted lithospheric plate detaches from the upper part and sinks into the mantle. The process was first hypothesized as a way to account for observed reversals of subduction polarity in ancient orogens (McKenzie 1969) and for gaps in the distributions of earthquake hypocentres in Benioff zones (Isacks \& Molnar 1969). Slab break-off is thought to have occurred or to be occurring in many places on Earth, including the New Hebrides arc (Pascal et al. 1973; Chatelain et al. 1992), the Sunda arc (Kundu \& Gahalaut 2011), eastern Turkey and the Caucasus (Faccenna et al. 2006; Zor 2011), the Hindu Kush (Schmalholz 2011), the European Alps (von
Blanckenburg \& Davies 1995; Regard et al. 2008), northern Central America (Rogers et al. 2002), the southern Banda arc (Buiter et al. 2002), southern Italy (Westaway 1993) and the Appenines (Wortel \& Spakman 1992). Yet the mechanism or mechanisms giving rise to break-off remain controversial.

Since the mid 1990s numerous geodynamic models of slab breakoff have been developed. These are of two types: thermomechanical and purely mechanical. Some of the earliest thermomechanical studies combined 2-D thermal models with parametrized representations of the strength distribution in the lithosphere (Davies \& von Blanckenburg 1995; Ton \& Wortel 1997). These studies focused on the detachment of oceanic lithosphere from continental lithosphere after continental collision, which is usually considered to be 


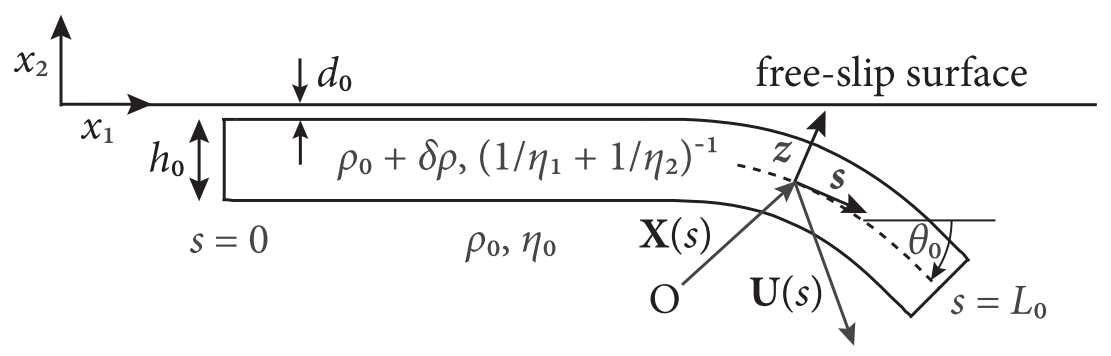

Figure 1. Definition sketch of the initial geometry of the model.

the main tectonic context in which slab break-off occurs. Larsen et al. (1995) studied 2-D time-dependent convection with nonNewtonian and temperature-dependent rheology, and found that slabs can break off after they are weakened by strong viscous dissipation. Li et al. (2002) proposed a simple dynamic model for the depth of continental subduction coupled with a thermal model for the slab strength, and concluded that slab break-off is favoured by the slowing down of convergence due to the resistance of the plate's continental portion to being subducted. Gerya et al. (2004) used a 2-D thermomechanical model to study slab necking after the cessation of active subduction, and found that necking occurs in a few Ma after a period of slab weakening by thermal diffusion. Andrews \& Billen (2009) studied 2-D thermomechanical models with composite Newtonian/non-Newtonian rheology, concluding that detachment requires a non-Newtonian upper mantle. They identified two different modes of detachment according to whether weakening of the slab is caused by warming or by strain softening. Burkett \& Billen (2010) used a 3-D thermomechanical model to study slab break-off after ridge-trench collision, focusing on how the detachment dynamics depend on the geometry of the ridge segments approaching the trench. Baumann et al. (2010) used a 2-D numerical model including constraints from petrology to explore the influence of slab age, convergence rate and phase transitions on the viscous mode of slab detachment. Using a 2D thermomechanical model, Duretz et al. (2012) quantified the depth and timing of slab breakoff and found that viscous necking is the dominant deformation process during slab detachment. Van Hunen \& Allen (2011) used 2-D and 3-D models with non-Newtonian rheology to quantify slab break-off following continental collision, and observed break-off in the form of propagating slab windows and tears. Finally, Bellas et al. (2018) used a 2-D thermal convection model with a vertical slab to study the influence of damage rheology and grain size evolution on necking instabilities.

While thermomechanical models are typically numerical in character, purely mechanical models lend themselves to a broader range of approaches including laboratory experiments, numerical simulations, and analytical solution. Laboratory studies of slab break-off often begin with a model oceanic lithosphere between model continents, and then compress the whole system by a moving wall driven by a piston (Faccenna et al. 2006; Regard et al. 2008). More recently, several researchers have begun to use novel strain-softening analogue materials in studies of slab break-off in forced oceanic subduction (Boutelier \& Cruden 2017) and free oceanic followed by continental subduction (Broerse et al. 2019). On the numerical side, Yoshioka \& Wortel (1995) constructed a 3-D finite-element model for a dipping tabular slab with a small initial tear on one side, and found that a strong stress concentration develops that may cause the tear to propagate laterally. von Tscharner et al. (2014) carried out 3D finite-element modeling of evolving break-off of non-Newtonian slabs, exploring both symmetric and asymmetric configurations.
Finally, Schmalholz (2011) proposed a zero-dimensional analytical model in the form of an ordinary differential equation for the temporal evolution of the minimum thickness of a necking slab with a power-law rheology, and compared the model's predictions with 2-D finite element calculations.

Many of the models just discussed are quite complicated, and it is not always easy to unravel the different dynamic effects at work and to identify those that are essential to slab break-off. Our aim here is to formulate a simpler, purely mechanical model that embodies a limited number of key physical mechanisms. In that sense, our work is very much in the spirit of Schmalholz (2011), who also emphasized the physical insight that comes from studying simple models. However, our model builds on his in several ways. First, it features a viscous sheet with a realistic shape comprising a flat plate pulled by the negative buoyancy of a curved downwarddipping slab. Secondly, the rheology of the sheet is assumed to be a realistic composite of Newtonian and non-Newtonian (shearthinning) components. Thirdly, the model allows deformation of the sheet by both stretching and bending. And finally, the model accounts for the interaction of the sheet with the flow in the ambient mantle. All of these physical effects are bundled into a single integral equation, the boundary-integral/thin-sheet (BITS) equation, which can be written down explicitly but must be solved numerically. Our approach is therefore a semi-analytical one.

\section{MODEL SETUP}

The initial geometry of the time-dependent model to be studied is shown in Fig. 1. A thin viscous sheet of length $L_{0}$ and uniform thickness $h_{0} \ll L_{0}$ is immersed in a half-space of fluid with a constant viscosity $\eta_{0}$ bounded above by a free-slip (mirror symmetry) surface. The horizontal portion ('plate') of the sheet is separated from the free-slip surface by a thin lubrication layer of uniform thickness $d_{0}$. The downward-dipping portion of the sheet (the 'slab') has length $l_{0}$, and its shape is given by eq. (1) of Ribe (2010). The initial dip of the leading edge of the slab is $\theta_{0}$. The viscosity of the sheet is the harmonic mean of a constant Newtonian viscosity $\eta_{1}$ and a nonNewtonian (shear thinning) viscosity $\eta_{2}$ that will be defined later. The sole driving force for the flow is the negative buoyancy $g \delta \rho$ of the sheet relative to the ambient fluid, where $g$ is the gravitational acceleration.

Because the sheet is thin, its dynamics can be described entirely in terms of quantities defined on its mid-surface, a portion of which is shown by the dashed line in Fig. 1. Let $s$ be the arclength along the mid-surface, $z$ be a coordinate normal to the mid-surface, and $\mathbf{s}$ and $\mathbf{z}$ the corresponding unit vectors. The Cartesian coordinates of a point $s$ on the mid-surface are $\mathbf{X}(s)$, and the fluid velocity on the mid-surface is $\mathbf{U}(s)$.

As the initial state shown in Fig. 1 evolves in time its geometry will change in several ways. Due to stretching of the sheet its length 
$L(t)$ will no longer be $L_{0}$, and its thickness $h(s, t)$ will no longer be constant. The thickness $d(s, t)$ of the lubrication layer will also depart from its initially constant value $d_{0}$. Finally, the slab's length $l(t)$ will increase continually, and the slab will no longer have a simple shape that can be described analytically.

\section{BOUNDARY-INTEGRAL/THIN-SHEET (BITS) MODEL}

To determine the flow in the system shown in Fig. 1, we shall use the hybrid BITS model proposed by Xu \& Ribe (2016). The model combines an asymptotic thin-layer description of the sheet and a boundary-integral description of the flow in the ambient fluid, and leads to the following integral equation for the mid-surface velocity $\mathbf{U}(s)$ :

$\mathbf{U}(s)=\frac{1}{\eta_{1}} \int_{0}^{L}\left[(\gamma-1) \mathbf{N}^{\prime}(p)+\gamma h(p) \delta \rho \mathbf{g}\right] \cdot \mathbf{J}(p, s) \mathrm{d} p$,

where $\mathbf{U}(s)=\mathbf{U}(\mathbf{X}(s)), \mathbf{J}(p, s)=\mathbf{J}(\mathbf{X}(p)-\mathbf{X}(s)), \gamma=\eta_{1} / \eta_{0}$, and a prime denotes a derivative with respect to arclength (the dummy variable of integration $p$ in this case) along the mid-surface. The vector $\mathbf{N}$ is called the stress resultant, and is defined as

$\mathbf{N}=\int_{-h / 2}^{h / 2}\left(\sigma_{s s} \mathbf{s}+\sigma_{s z} \mathbf{z}\right) \mathrm{d} z \equiv N \mathbf{s}+Q \mathbf{z}$

where $\sigma_{s s}$ and $\sigma_{s z}$ are, respectively, the normal (fibre) stress and the shear stress acting on cross-sections of the sheet. For future reference, we note that torque balance on the sheet requires $Q=$ $M$, where a prime denotes differentiation with respect to $s$ and the bending moment $M$ is

$M=\int_{-h / 2}^{h / 2} z \sigma_{s s} \mathrm{~d} z$.

The tensor $\mathbf{J}$ in (1) is the Green function representing the flow due to a point force (Stokeslet) in a fluid half-space bounded above by a free-slip surface. It has the general form

$J_{\alpha \beta}(\mathbf{y}-\mathbf{x})=J_{\alpha \beta}^{*}(\mathbf{y}-\mathbf{x})+(-1)^{\beta+1} J_{\alpha \beta}^{*}\left(\mathbf{y}-\mathbf{x}^{I M}\right)$,

where $J_{\alpha \beta}^{*}$ is the Green's function for the flow due to a point force in an infinite fluid, $\mathbf{x}^{I M} \equiv \mathbf{x}-2 x_{2} \mathbf{e}_{2}$ is the mirror image of $\mathbf{x}$ above the free-slip surface, and the indices $\alpha$ and $\beta$ take on the values 1 or 2 . However, to avoid the singularity of the Green's function when $p=s$ we adopt the regularized Stokeslet of Cortez (2002), which represents the flow in an infinite fluid generated by a force distributed over a circle (in 2-D) of characteristic radius $\epsilon$. The explicit form of this Green's function is

$J_{\alpha \beta}^{*}(\mathbf{r})=\frac{1}{4 \pi}\left[-\delta_{\alpha \beta} \ln (R+\epsilon)+\frac{R+2 \epsilon}{R(R+\epsilon)}\left(\epsilon \delta_{\alpha \beta}+\frac{r_{\alpha} r_{\beta}}{R+\epsilon}\right)\right],(5)$

where $R=\sqrt{|\mathbf{r}|^{2}+\epsilon^{2}}$. We found by trial and error that the value $\epsilon$ $=0.05 h_{0}$ gives the best (error $<3$ per cent) agreement of the BITS prediction with that of an 'exact' boundary-element approach using unregularized Stokeslets (Ribe 2010) for a simple model problem of a flat $\left(\theta_{0}=0\right)$ sheet with $L_{0} / h_{0}=4, \gamma=10^{4}$, and $d_{0} / h_{0} \in[0.1$, 0.6]. We shall therefore use $\epsilon=0.05 h_{0}$ henceforth.

The BITS eq. (1) governs the instantaneous mid-surface velocity field $\mathbf{U}(s)$ for a sheet with a given mid-surface geometry $\mathbf{X}(s)$ and thickness $h(s)$. However, as the system evolves in time both $\mathbf{X}(s)$ and $h(s)$ will change according to the kinematic equations

$\frac{\mathrm{DX}}{\mathrm{D} t}=\mathbf{U}, \quad \frac{\mathrm{D} h}{\mathrm{D} t}=-h \mathbf{U}^{\prime} \cdot \mathbf{s}$, where $\mathrm{D} / \mathrm{D} t$ is a convective time derivative that follows material points on the mid-surface, the prime denotes differentiation with respect to $s$, and $\mathbf{U}^{\prime} \cdot \mathbf{s}$ is the rate of stretching of the mid-surface.

\section{NEWTONIAN REFERENCE MODELS}

The aim of this section is to obtain some preliminary physical insight by solving the BITS equations for the relatively simple case of a Newtonian sheet with constant viscosity $\eta_{1}$. The fibre stress resultant and bending moment for this case are

$N=4 \eta_{1} h \Delta, \quad \Delta=\mathbf{U}^{\prime} \cdot \mathbf{s}$,

$M=-\frac{1}{3} \eta_{1} h^{3} \dot{K}, \quad \dot{K}=\left(\mathbf{U}^{\prime} \cdot \mathbf{z}\right)^{\prime}$,

where $\Delta$ is rate of stretching of the mid-surface and $\dot{K}$ is the rate of change of its curvature. We solved the BITS momentum equation at each time step using a discrete Lagrangian formulation (Appendix D). The evolution equation for $\mathbf{X}$ was then solved using forward Euler time stepping. Finally, the sheet's thickness was updated using the fact that the area (thickness times length) of each Lagrangian element of the sheet remains constant. The initially uniform grid spacing on the midsurface was $0.067 h_{0}$, and the time step was calculated so that no material point on the mid-surface moved more than a distance $0.05 h_{0}$ in one time step.

\subsection{Instantaneous solutions}

We begin by considering instantaneous solutions of the BITS momentum equation. Because inertia is negligible, the evolutionary history of a subducting sheet is a sequence of quasi-static configurations whose dynamics are determined entirely by the sheet's instantaneous shape. It therefore makes sense first to study the instantaneous dynamics of the sheet without the added complexity of the purely kinematic time evolution. To do this, we interpret the model parameters in Fig. 1 not as initial values of a time-dependent simulation, but rather as free parameters that can be varied to represent a wide range of different sheet shapes at some arbitrary instant in time.

Following Ribe (2010), we anticipate that the vertical speed $V>$ 0 of the leading end of the sinking slab should satisfy the scaling law

$\frac{V}{V_{\text {Stokes }}}=\mathrm{fct}(\mathrm{St}), \quad V_{\text {Stokes }}=\frac{h_{0} l_{0} g \delta \rho}{\eta_{0}}, \quad \mathrm{St}=\frac{\eta_{1}}{\eta_{0}}\left(\frac{h_{0}}{l_{b}}\right)^{3}$.

Here $V_{\text {Stokes }}$ is the characteristic Stokes sinking speed of a tabular body of thickness $h_{0}$ and lateral dimension $l_{0}$ in a fluid of viscosity $\eta_{0}$, and St is the 'flexural stiffness' of the sheet. The length $l_{b}$ is the 'bending length', that is the length of the portion of the sheet where deformation is predominantly by bending. In geophysical terms $l_{b}$ is the sum of the slab length and the lateral extent of the flexural bulge seaward of the trench. For each numerical solution of the BITS momentum equation, $l_{b}$ is calculated as shown in fig. 5 of Ribe (2010).

Fig. 2(a) shows 44 numerically predicted pairs (St, $V / V_{\text {Stokes }}$ ) for $\theta_{0}=60^{\circ}, d_{0} / h_{0}=0.2$ and different values of $\gamma$ and $l_{0} / h_{0}$. All solutions collapse onto a universal curve with two distinct limits. In the 'Stokes' limit, the bending resistance of the sheet is negligible, and the sinking speed is controlled entirely by the viscosity $\eta_{0}$ 


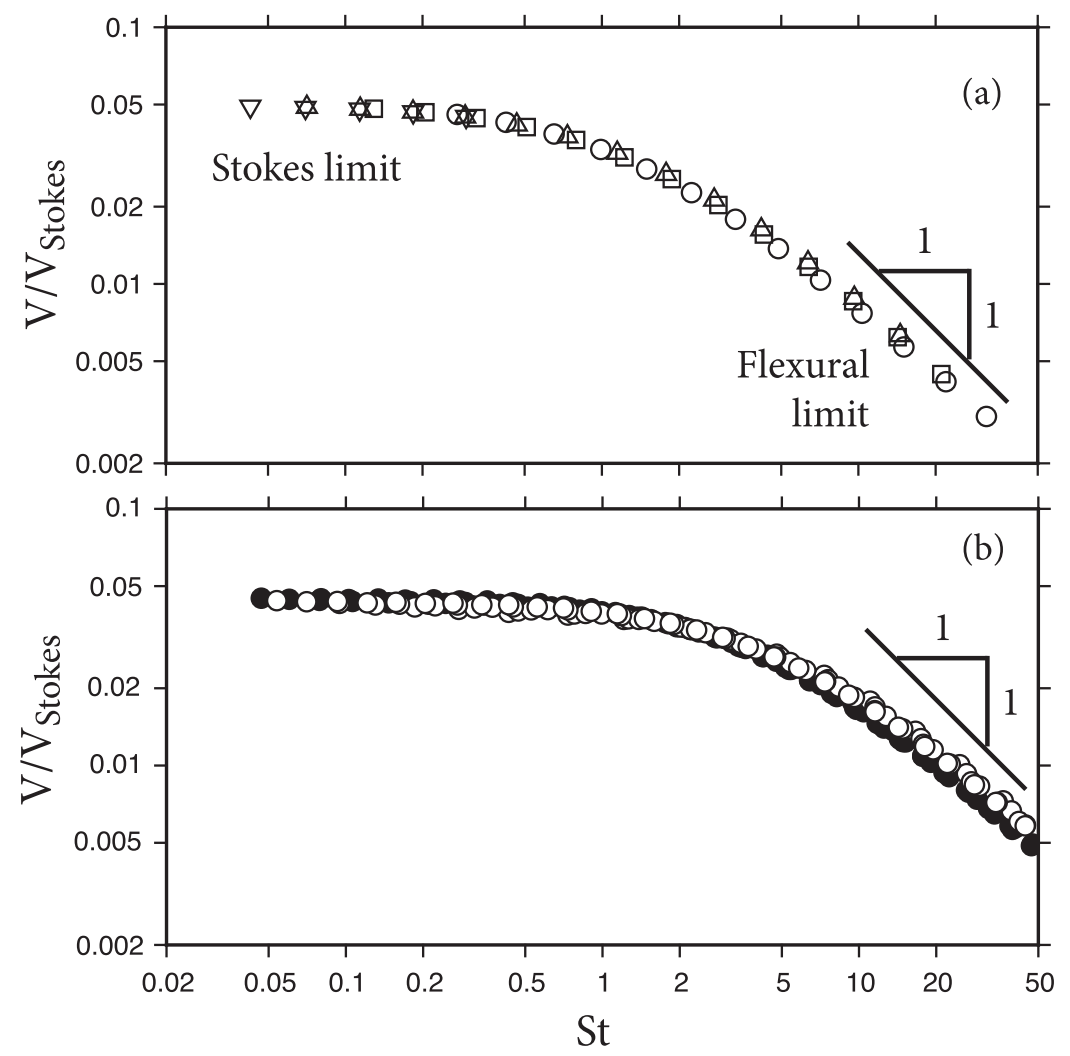

Figure 2. Scaling of the instantaneous vertical sinking speed $V$ of a freely subducting Newtonian sheet predicted by (a) the BITS model and (b) full 2-D boundary-element simulation (Ribe 2010). The Stokes velocity scale $V_{\text {Stokes }}$ and the flexural stiffness $S t$ are defined by (8). In part (a) the symbols represent solutions of the BITS equation for $\theta_{0}=60^{\circ}, d_{0} / h_{0}=0.2, \gamma \in[32,32000]$ and $l_{0} / h_{0}=4$ (circles), 6 (squares), 8 (triangles), and 10 (inverted triangles). In part (b), $\theta_{0}=60^{\circ}, \gamma \in[31.6,100000], l_{0} / h_{0} \in[4,10]$ and $d_{0} / h_{0}=0.1$ (open circles) or 0.2 (filled circles).

of the ambient fluid. In the 'flexural' limit, by contrast, bending resistance is dominant and the sinking speed is controlled entirely by the sheet's viscosity $\eta_{1}$. The slope of the curve in this limit is predicted by the scaling analysis of Ribe (2010) to be -1 .

For comparison, Fig. 2 b shows $V / V_{\text {Stokes }}$ vs. St as predicted by full boundary-element method (BEM) simulation using unregularized Stokeslets (Ribe 2010). The BITS and BEM predictions agree to within about 10 per cent in the Stokes limit. However, in the flexural limit the BEM predicts sinking speeds that exceed the BITS predictions by about a factor of 2 . The reason for this discrepancy is unclear. However, we note that BITS does a better job at predicting the expected slope of the curve in the flexural limit. This fact, together with the tight collapse of the numerical data in Fig. 2(a), gives us confidence in the applicability of the BITS model.

\subsection{Temporal evolution}

We now turn to the temporal evolution of subducting Newtonian sheets. Fig. 3 shows this evolution for $\gamma=100$ (upper right) and 1000 (lower left), starting from an initial condition (dashed lines) that is the same in both cases. The subsequent shapes of the sheet are shown at times when the depths of the slab's leading end (measured from the upper surface of the plate) are $5 h_{0}$ and $10 h_{0}$. The slab with the lower bending resistance $(\gamma=100)$ sinks almost vertically. By contrast, the stiffer slab $(\gamma=1000)$ 'bends over backwards' because its strong bending resistance makes it hard to unbend. Unbending will of course eventually occur, but only when the slab has reached significantly greater depths (Xu \& Ribe 2016).

\section{NON-NEWTONIAN SHEETS}

Eq. (1) was derived for a Newtonian sheet with constant viscosity $\eta_{1}$. However, it can easily be recast in a form suitable for a nonNewtonian sheet by taking the stiff-sheet limit $\gamma \gg 1$, whereupon we obtain

$\mathbf{U}(s)=\frac{1}{\eta_{0}} \int_{0}^{L}\left[\mathbf{N}^{\prime}(p)+h(p) \delta \rho \mathbf{g}\right] \cdot \mathbf{J}(p, s) \mathrm{d} p$.

The stress resultant $\mathbf{N}$ can now be replaced by an explicit form for a desired non-Newtonian rheology, at which point (9) describes the buoyancy-driven motion of a non-Newtonian sheet in an ambient Newtonian fluid with viscosity $\eta_{0}$.

The numerical solution of the non-Newtonian BITS equation, like that of its Newtonian counterpart, is based on the notion of the Rayleigh dissipation potential (RDP), from which the viscous forces within the sheet can be obtained by derivation with respect to the velocity. To introduce the RDP, we begin by recalling that the components of the strain rate tensor within a thin sheet having any rheology are

$e_{z s}=0, \quad e_{s s}=-e_{z z}=\Delta-\dot{K} z$.

The viscosity of the sheet is

$\eta=\left(\frac{1}{\eta_{1}}+\frac{1}{\eta_{2}}\right)^{-1}, \quad \eta_{2}=B_{n} I^{1 / n-1}$,

where $\eta_{1}$ is a constant Newtonian viscosity, $B_{n}$ is a constant powerlaw stiffness, and

$I=\left(\frac{e_{i j} e_{i j}}{2}\right)^{1 / 2} \equiv|\Delta-\dot{K} z|$. 


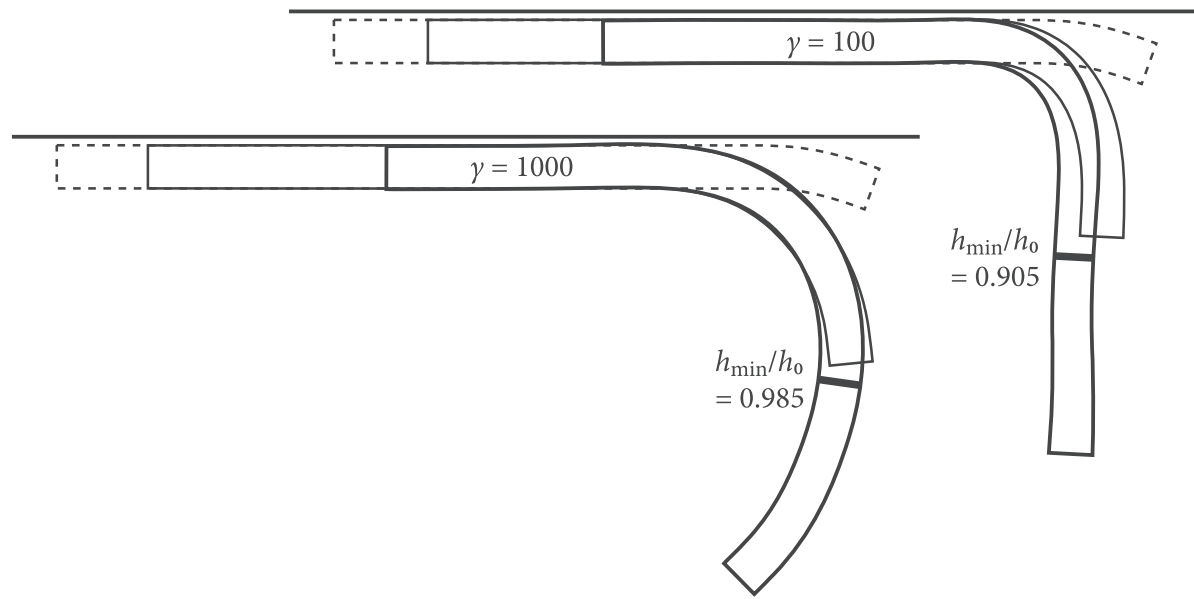

Figure 3. Temporal evolution of Newtonian sheets with viscosity ratios $\gamma=100$ (upper right) and 1000 (lower left). The initial condition (dashed lines) is the same in both cases, and has $l_{0} / h_{0}=3, L_{0} / h_{0}=19$ and $d_{0} / h_{0}=0.2$.

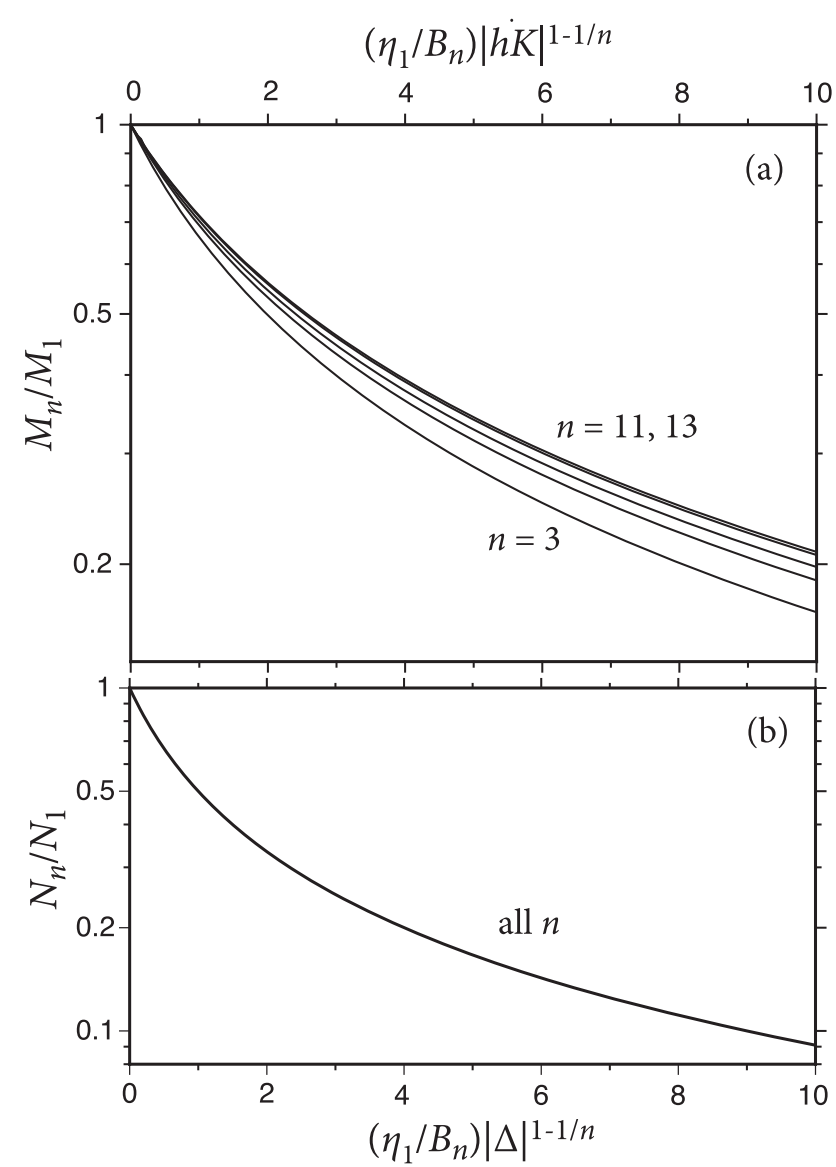

Figure 4. Weakening effect of composite rheology in deformation by (a) pure bending and (b) pure stretching. $M_{n}$ and $N_{n}$ are the bending moment and stress resultant, respectively, for a sheet with a composite rheology with a power-law index $n$, where $n=1$ corresponds to the Newtonian limit. In part (a), results are shown for $n=3$ to 13 in steps of 2 .

The fibre stress within the sheet is

$\sigma_{s s}=4 \eta(\Delta-\dot{K} z)=4(\Delta-\dot{K} z)\left(\frac{1}{\eta_{1}}+\frac{|\Delta-\dot{K} z|^{1-1 / n}}{B_{n}}\right)^{-1}$.
Finally, we define the local (not yet integrated along the mid-surface) RDP as

$\phi=\frac{1}{2} \int_{-h / 2}^{h / 2} e_{s s} \sigma_{s s} \mathrm{~d} z$.

The integral (14) is evaluated in Appendix A, where an explicit expression is given for $n=3$.

An important dimensionless group is the characteristic ratio $\eta_{1} / \eta_{2}$ of the Newtonian to the non-Newtonian viscosity. Introducing the scale $\Delta-\dot{K} z \sim h_{0} g \delta \rho / \eta_{1}$, we find

$\frac{\eta_{1}}{\eta_{2}} \sim \frac{\eta_{1}}{B_{n}}\left(\frac{h_{0} g \delta \rho}{\eta_{1}}\right)^{1-1 / n} \equiv \lambda$.

Greater values of $\lambda$ correspond to more strongly non-Newtonian behaviour. In our numerical simulations we use $0.45 \leq \lambda \leq 4.5$, which is a reasonable range based on estimates from laboratory rheological data (Appendix B). For a purely Newtonian sheet the parameter $\lambda$ is irrelevant, and the only dimensionless group is the viscosity ratio $\gamma=\eta_{1} / \eta_{0}$.

\subsection{Weakening effect of composite rheology in pure bending and stretching deformations}

To illustrate the weakening effect of composite rheology, we consider bending and stretching separately. Consider first pure bending, for which the viscosity is given by (11) with $I=|\dot{K} z|$. The fibre stress is then $\sigma_{s s}=-4 \eta \dot{K} z$. The weakening effect of composite rheology can be quantified by the ratio of the bending moment $M_{n}$ for a sheet with a composite rheology to the bending moment $M_{1}=-\eta_{1} \dot{K} h^{3} / 3$ of a purely Newtonian sheet, which is

$\frac{M_{n}}{M_{1}}=24 \int_{0}^{1 / 2} \frac{\zeta^{2}}{1+\lambda_{b} \zeta^{1-1 / n}} \mathrm{~d} \zeta$

Here $\lambda_{b}=\left(\eta_{1} / B_{n}\right)(|\dot{K}| h)^{1-1 / n}$ is a dimensionless group that vanishes for a purely Newtonian sheet. As an explicit example,

$\frac{M_{3}}{M_{1}}=-\frac{362^{2 / 3}}{\lambda_{b}^{4}}+\frac{12}{\lambda_{b}^{3}}-\frac{182^{1 / 3}}{5 \lambda_{b}^{2}}+\frac{92^{2 / 3}}{7 \lambda_{b}}+\frac{72}{\lambda_{b}^{9 / 2}} \tan ^{-1}\left(\frac{\lambda_{b}^{1 / 2}}{2^{1 / 3}}\right)$,

where $\lambda_{b}=\left(\eta_{1} / B_{3}\right)(|\dot{K}| h)^{2 / 3}$. 
For pure stretching deformation, the viscosity is given by (11) with $I=|\Delta|$. The weakening effect of composite rheology is characterized by the ratio $N_{n} / N_{1}$, where the fibre stress resultant $N$ is defined by (2). We find

$\frac{N_{n}}{N_{1}}=\frac{1}{1+\lambda_{s}}$

for all $n$, where $\lambda_{s}=\left(\eta_{1} / B_{n}\right)|\Delta|^{1-1 / n}$.

Fig. 4(a) shows $M_{n} / M_{1}$ as a function of $\lambda_{b}$ for $n=3-13$, while Fig. 4(b) shows $N_{n} / N_{1}$ as a function of $\lambda_{s}$ for all $n$. Both $M_{n} / M_{1}$ and $N_{n} / N_{1}$ decrease monotonically as functions of $\lambda_{b}$ or $\lambda_{s}$, showing that a sheet with a composite rheology is significantly weaker than a Newtonian sheet in both bending- and stretching-dominated deformations.

\subsection{Scaling of the sinking speed}

Following the order in which we presented our Newtonian reference cases, we now consider the instantaneous vertical sinking speed $(V)$ of a non-Newtonian sheet. We begin with dimensional analysis. The nine quantities on which $V$ depends are $h_{0}, d_{0}, l_{0}, \theta_{0} g \delta \rho, \eta_{1}, \eta_{0}$, $B_{n}$ and $n$. The length $L_{0}$ of the sheet has only a small effect on $V$ (Ribe 2010) and is therefore omitted from the foregoing list. Of the ten quantities listed, three have independent dimensions. According to Buckingham's $\Pi$-theorem, therefore, seven independent dimensionless combinations of the original quantities can be formed. We therefore have

$\frac{V}{V_{\text {Stokes }}}=\mathrm{fct}\left(\frac{l_{0}}{h_{0}}, \frac{d_{0}}{h_{0}}, \theta_{0}, \gamma, \lambda, n\right)$,

where $\lambda$ is defined by (15). The unknown function on the righthand side of (19) has six arguments, and would be very difficult to characterize using numerical solutions of the BITS equation. However, a much simpler result can be obtained by means of a scaling analysis of the forces acting on the sheet. The relevant portion of the sheet to consider is the bending length $l_{b}$ introduced earlier. Three forces (per unit length along the strike of the trench) act on this portion of the sheet: the gravitational driving force $F_{\text {grav }}$, the integral $F_{\text {ext }}$ of the tractions applied by the exterior flow and the internal viscous force $F_{\text {int }}$ due to the sheet's inherent resistance to bending. The gravitational force is $F_{\text {grav }} \sim h_{0} l_{0} g \delta \rho$. The external viscous traction per unit area is $\sigma_{\text {ext }} \sim \eta_{0} V / l_{b}$, whence $F_{\text {ext }} \sim \eta_{0} V$. Finally, the internal viscous force $F_{\text {int }}$ is just the resultant $Q$ of the vertical shear stress acting on a cross-section of the sheet at the seaward end of the flexural bulge. Torque balance implies $Q=M^{\prime} \sim$ $M / l_{b}$, where $M$ is the bending moment and the arcwise derivative has a typical scale $l_{b}^{-1}$. We previously found that the bending moment for a sheet with a composite rheology is

$M_{n}=M_{1} \alpha_{n}\left(\frac{\eta_{1}}{B_{n}}\left|h_{0} \dot{K}\right|^{1-1 / n}\right) \sim \eta_{1} h_{0}^{3} \dot{K} \alpha_{n}\left(\frac{\eta_{1}}{B_{n}}\left|h_{0} \dot{K}\right|^{1-1 / n}\right)$,

where the functions $\alpha_{n}$ are just the curves in Fig. 4(a). Now set $\dot{K} \sim V / l_{b}^{2}$ in the prefactor to the function $\alpha_{n}$, and replace $\left|h_{0} \dot{K}\right|$ by $\left|h_{0} \dot{K}\right|_{\max }$. The internal viscous force is then

$F_{\text {int }} \sim \eta_{1}\left(\frac{h_{0}}{l_{b}}\right)^{3} V \alpha_{n}\left(\frac{\eta_{1}}{B_{n}}\left|h_{0} \dot{K}\right|_{\max }^{1-1 / n}\right)$.

Now the force balance $F_{b} \sim F_{\text {ext }}$ yields

$V \sim \frac{h_{0} l_{0} g \delta \rho}{\eta_{0}} \equiv V_{\text {Stokes }}$

Thus we expect $V=V_{\text {Stokes }} \mathrm{fct}\left(F_{\text {int }} / F_{\text {ext }}\right)$, or

$\frac{V}{V_{\text {Stokes }}}=\mathrm{fct}\left(\mathrm{St}^{\mathrm{red}}\right), \quad \mathrm{St}^{\mathrm{red}}=\operatorname{St} \alpha_{n}\left(\gamma^{1-1 / n} \lambda|\hat{\dot{K}}|_{\max }^{1-1 / n}\right)$.

In (23), $|\hat{\dot{K}}|_{\max }=\left(\eta_{0} / g \delta \rho\right)|\dot{K}|_{\max }$, St is the Newtonian flexural stiffness defined by (8) and $\mathrm{St}^{\text {red }}$ is a reduced stiffness that takes into account the weakening effect of the composite rheology.

If the foregoing scaling analysis is correct, numerical solutions of the BITS equation for a fixed value of $\theta_{0}$ and any value of $n$ should collapse onto a universal curve in a plot of $V / V_{\text {Stokes }}$ versus $\mathrm{St}^{\text {red }}$. To test this, we determine points $\left(\mathrm{St}^{\mathrm{red}}, V / V_{\text {Stokes }}\right)$ by solving the BITS equation for $n=3, \theta_{0}=60^{\circ}, l_{0} / h_{0}=4$, four values of $\gamma \in[100,3160]$ and $\lambda$ up to a different maximum value for each value of $\gamma$. The results are shown by the four thick lines in Fig. 5, together with the universal curve from Fig. 2 (thin line). The thick lines follow the thin line almost exactly, demonstrating the validity of our scaling law (23).

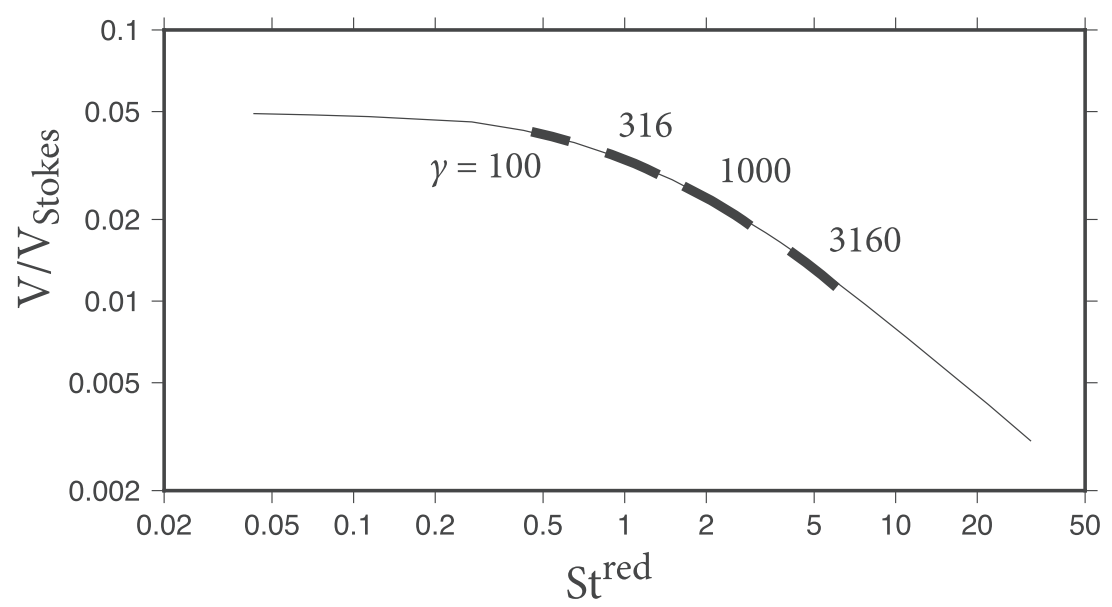

Figure 5. Scaling of the instantaneous vertical sinking speed $V$ of a freely subducting non-Newtonian sheet with $n=3$. The four short thick lines are for $d_{0} / h_{0}$ $=0.2, \theta_{0}=60^{\circ}, l_{0} / h_{0}=4$ and the values of $\gamma$ indicated. $\lambda$ increases from right to left along each thick line, up to maximum values 0.46 (for $\gamma=100$ and 316), 0.42 (for $\gamma=1000$ ) and 0.23 (for $\gamma=3160$ ). The reduced stiffness $\mathrm{St}^{\mathrm{red}}$ is defined by (23). The thin line is the universal curve from Fig. 2. 

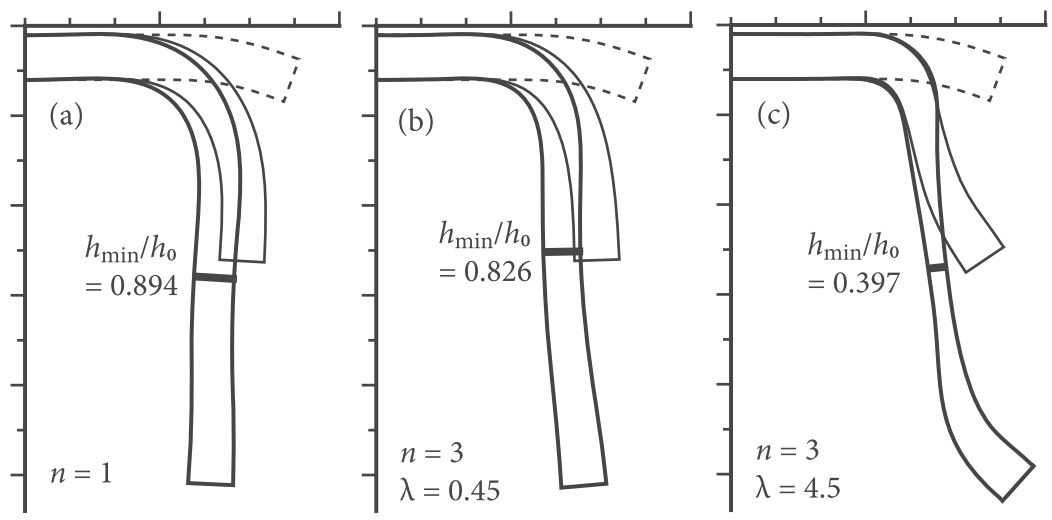

Figure 6. Evolution of free subduction of Newtonian and non-Newtonian sheets with a viscosity ratio $\gamma=100$. (a) $n=1$ (Newtonian reference case); (b) $n$ $=3$ and $\lambda=0.45$; (c) $n=3$ and $\lambda=4.5$. The initial condition (dashed lines) has $l_{0} / h_{0}=3, L_{0} / h_{0}=19$ and $d_{0} / h_{0}=0.2$. The minimum thickness of the slab when its leading end is at depth $10 h_{0}$ is indicated by the heavy black lines.
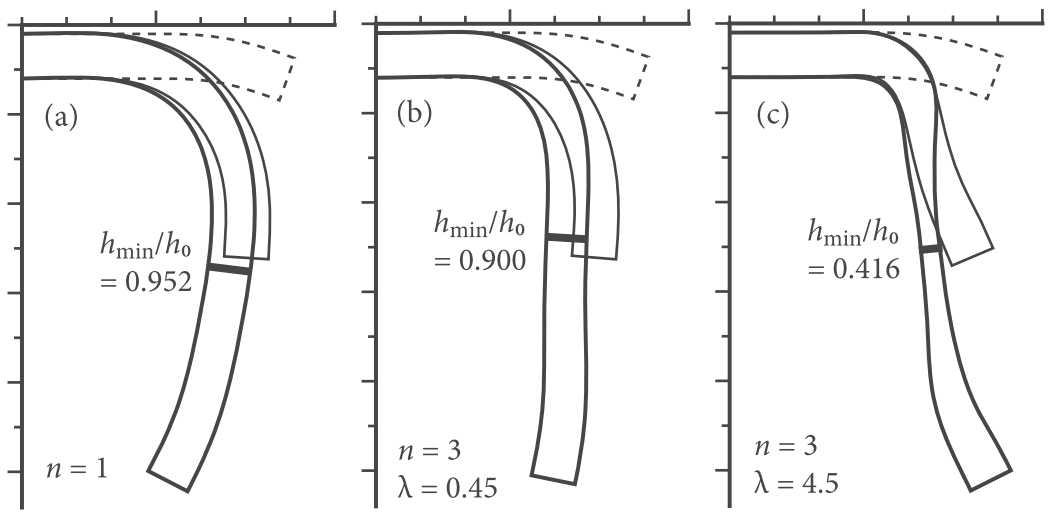

Figure 7. Same as Fig. 6, but for $\gamma=316$.
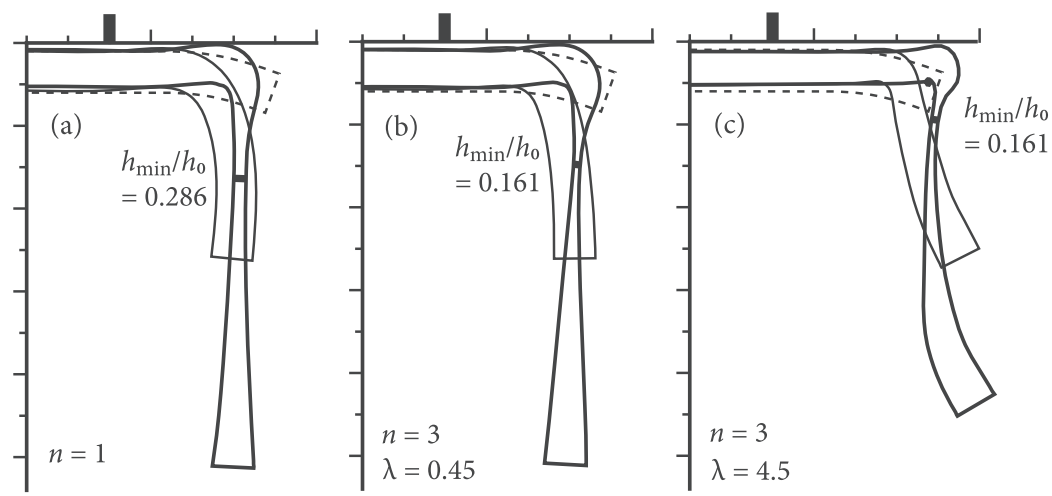

Figure 8. Arrested subduction of (a) Newtonian and (b, c) non-Newtonian sheets with viscosity ratio $\gamma=100$. The initial condition is the same as in Fig. 6 , except that the buoyant continental portion of the plate initially to the left of the vertical bar has a negative density anomaly $\delta \rho_{\text {cont }}=-3 \delta \rho$.

\subsection{Temporal evolution of non-Newtonian subduction}

We now turn to the temporal evolution of subducting sheets with composite diffusion/dislocation creep rheology. We first consider the case of 'free' subduction in which the subducting slab freely pulls an attached negatively buoyant plate. Fig. 6 shows snapshots of the subduction history for a viscosity contrast $\gamma=100$ and three combinations of values of $n$ and $\lambda$ : (a) a Newtonian sheet $(n=1)$, (b) a non-Newtonian sheet with $n=3$ and $\lambda=0.45$ and (c) a nonNewtonian sheet with $n=3$ and $\lambda=4.5$. While significant thinning of the slab occurs in the non-Newtonian cases, it is not sufficient to cause slab break-off, even for $\lambda=4.5$. For $\lambda=4.5$ the lowermost portions of the two slabs shown are bent counter-clockwise relative to the vertical. This is a consequence of the drag applied to the slabs by the 'mantle wind' flowing counter-clockwise around their tips.

Fig. 7 is the same as Fig. 6 but for a stiffer slab with $\gamma=316$. Again, significant thinning of the slab occurs, but not enough for break-off. We tentatively conclude that it is difficult to get slab break-off to occur when subduction is free.

We now turn to 'arrested' subduction, wherein subduction is slowed or stopped when a positively buoyant (continental) portion of the plate reaches the trench. To model this behaviour, we suppose 
that the initial density anomaly of the sheet is

$\delta \rho=\frac{1}{2}\left[\delta \rho_{0}+\delta \rho_{c}+\left(\delta \rho_{0}-\delta \rho_{c}\right) \tanh \left(\frac{s-s_{c}}{\chi h_{0}}\right)\right]$.

eq. (24) states that $\delta \rho=\delta \rho_{c}$ to the left of the arclength $s=s_{c}$ and $\delta \rho=\delta \rho_{0}$ to the right of it, with a transition region of width $\chi h_{0}$ between the two domains. In the sequel we shall assume the (admittedly somewhat arbitrary) values $\delta \rho_{\text {cont }}=-3 \delta \rho_{0}, s_{c}=15 h_{0}$ and $\chi=0.3$. Since the length $L_{0}-l_{0}$ of our plates is $16 h_{0}$, the position $s_{c}=15 h_{0}$ is a distance $h_{0}$ to the left of the initial position of the trench. This allows for a short period of oceanic subduction before the buoyant continent arrives at the trench.

Fig. 8 shows the evolution of arrested subduction for a viscosity ratio $\gamma=100$. Slab thinning is significantly greater than for free subduction, and thinning has progressed nearly to break-off in the non-Newtonian cases with $\lambda=0.45$ and 4.5. In Fig. 8(c) the counterclockwise bending of the slabs due to the mantle wind around their tips is evident, as it was in Fig. 6(c).

The results for arrested subduction with $\gamma=316$ differ only quantitatively from those in Fig. 8, and are not shown to save space. The overall conclusions are that arrested subduction strongly favours slab break-off, and that it pushes the point of maximum thinning to shallower depths relative to the free subduction case.

\section{1-D MODEL FOR SLAB BREAK-OFF}

The striking difference in the degree of slab thinning between free (Figs 6 and 7) and arrested (Fig. 8) subduction can be explained using a simple 1-D 'viscous dripping' model in which the slab is vertical and deforms by stretching only, without bending. Our starting point is the model of Wilson (1988), who considered the slow dripping of a viscous fluid ejected downward at a constant volumetric rate from a circular nozzle. Our model extends Wilson's in three ways: we replace his axisymmetric jet with a 2-D sheet; we allow the ejection rate to be variable; and we suppose that the fluid has a composite Newtonian/non-Newtonian rheology. For concision we shall refer to this as the 'DRIP' model.

The model geometry is shown in Fig. 9(a). Slab material is ejected downward at a variable speed $U_{0}(t)$ from a slot of width $h_{0}$ that represents the trench. Following Wilson (1988), we use a Lagrangian coordinate system in which cross-sections of the slab are labelled by the time $\tau$ at which they emerged from the slot (Fig. 9a). Thus $\tau$ $=0$ at the bottom end of the slab, while $\tau=t$ on the cross-section emerging at the present instant $t$. Let $Z(\tau, t)$ be the distance below the slot at time $t$ of a cross-section that emerged at time $\tau$, and let $h(\tau, t)$ be the width of that cross-section. Consider an element of volume contained between cross-sections that emerged between $\tau$ and $\tau+\mathrm{d} \tau$, and let the distances of these cross-sections below the slot be $Z$ and $Z+\mathrm{d} Z$. The area of the element is $h \mathrm{~d} Z$. This area must equal the area that originally exited the slot at velocity $U_{0}(\tau)$ at the earlier time $t=\tau$. Thus we have $-U_{0}(\tau) h_{0} \mathrm{~d} \tau=h \mathrm{~d} Z$, where the negative sign is present because $\tau$ increases upward while $Z$ increases downward. Therefore

$h \frac{\partial Z}{\partial \tau}=-h_{0} U_{0}(\tau)$.

Next, consider the force balance on the fluid between $\tau$ and $\tau+\mathrm{d} \tau$, and denote the longitudinal stress by $\Sigma(\tau, t)$. The net viscous force on the element must be balanced by its weight, which requires

$(\Sigma h)_{\tau}-(\Sigma h)_{\tau+\mathrm{d} \tau}-\delta \rho g h \mathrm{~d} Z=0$ or

$\frac{\partial}{\partial \tau}(\Sigma h)=-\delta \rho h \frac{\partial Z}{\partial \tau}=\delta \rho g h_{0} U_{0}(\tau)$.

Eq. (27) can be integrated subject to the condition $\Sigma=0$ at $\tau=0$ to yield

$\Sigma h=\delta \rho g h_{0} F(\tau)$.

where

$F(\tau)=\int_{0}^{\tau} U_{0}(\zeta) \mathrm{d} \zeta$

Eq. (28) states that the longitudinal force at a station $\tau$ equals the weight of all the fluid below that point.

The final step is to specify the constitutive relation for $\Sigma$, which is

$\Sigma=4 \eta_{1} \Delta\left(1+\frac{\eta_{1}}{B_{n}}|\Delta|^{1-1 / n}\right)^{-1}, \quad \Delta=-\frac{1}{h} \frac{\partial h}{\partial t}$,

where $\Delta$ is the rate of stretching of the sheet.

The foregoing equations can be combined to give

$-4 \eta_{1} \dot{h}\left[1+\frac{\eta_{1}}{B_{n}}(-\dot{h} / h)^{1-1 / n}\right]^{-1}=\delta \rho g h_{0} F(\tau)$,

where $\dot{h}=\partial h / \partial t$. It is helpful to write (25) and (31) in alternate forms in which each term has no dimensions. For this purpose, introduce time and distance scales

$t_{0}=\left(\frac{4 \eta_{1}}{g \delta \rho U_{s}}\right)^{1 / 2}, \quad Z_{0}=\left(\frac{4 \eta_{1} U_{s}}{g \delta \rho}\right)^{1 / 2} \equiv U_{s} t_{0}$,

where $U_{s}$ is a characteristic scale for the function $U_{0}(t)$. For $\eta_{1}=$ $10^{23} \mathrm{~Pa} \mathrm{~s}, \delta \rho=80 \mathrm{~kg} \mathrm{~m}^{-3}$ and $U_{s}=6 \mathrm{~cm} \mathrm{yr}^{-1}, t_{0}=16.4 \mathrm{Ma}$ and $Z_{0}=980 \mathrm{~km}$.

Using the scales (32), we can write (25) and (31) as

$\frac{t_{0}}{h_{0} Z_{0}} h \frac{\partial Z}{\partial \tau}=-\frac{U_{0}(\tau)}{U_{s}}$,

$\frac{t_{0}}{h_{0}} \dot{h}+\left[1+\Lambda\left(-t_{0} \dot{h} / h\right)^{1-1 / n}\right] \frac{F(\tau)}{U_{s} t_{0}}=0$,

where

$\Lambda=\frac{\eta_{1}}{B_{n}} t_{0}^{1 / n-1} \equiv \frac{\eta_{1}}{B_{n}}\left(\frac{g \delta \rho U_{s}}{4 \eta_{1}}\right)^{(n-1) / 2 n}$.

The dimensionless parameter $\Lambda$ measures the importance of the shear-thinning component of the rheology relative to the Newtonian component. Fluid dynamicists will recognize the procedure used to write down (33)-(35) as equivalent to a non-dimensionalization of the governing equations. We have chosen instead to use dimensional variables to aid readers who may not be familiar with non-dimensionalization.

\subsection{Analytical solution for a Newtonian slab}

Eqs (33) and (34) can be solved analytically for a Newtonian sheet with a constant convergence rate $U_{0}$. Setting $\Lambda=0, F(\tau)=U_{0} \tau$ and $U_{s}=U_{0}$, we obtain the following simplified form of (34):

$\dot{h}=-\frac{h_{0} \tau}{t_{0}^{2}}$.

Integrating (36) subject to the initial condition $h(t=\tau)=h_{0}$, we obtain

$h=h_{0}\left[1+\frac{\tau(\tau-t)}{t_{0}^{2}}\right]$. 
(a)

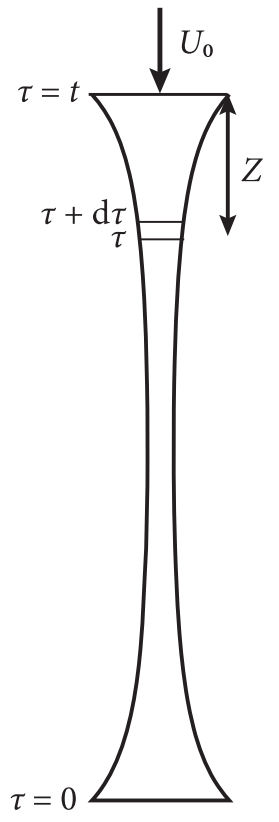

(b)
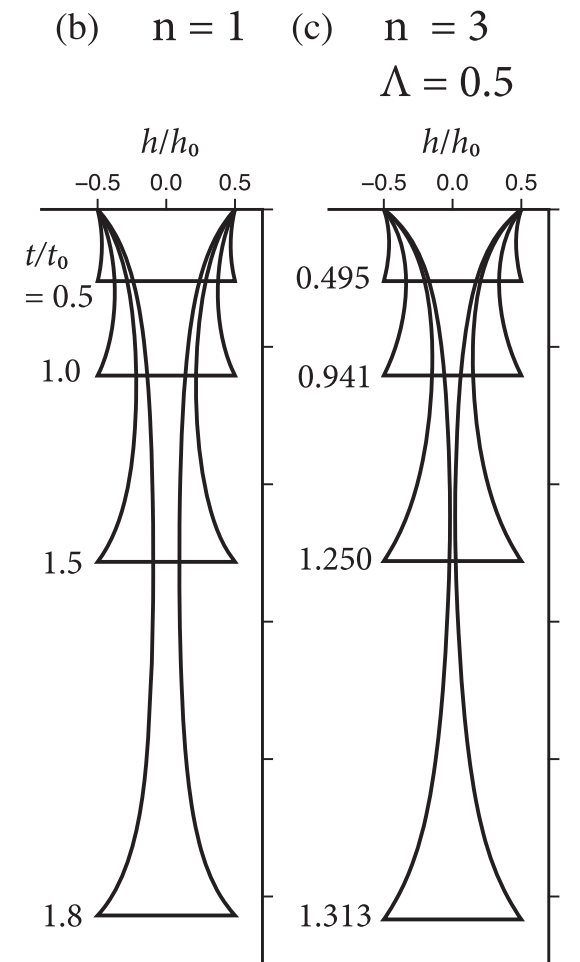

1.8 (c) $\mathrm{n}=3$

$$
\Lambda=0.5
$$

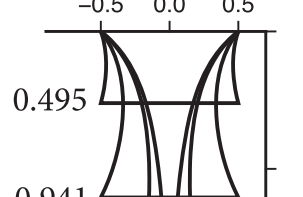

0.941

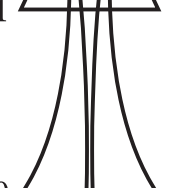

1.250

1.313 (d) $\mathrm{n}=3$

$\Lambda=1.5$

$h / h_{0}$

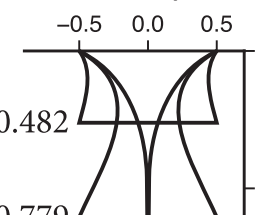

0.779
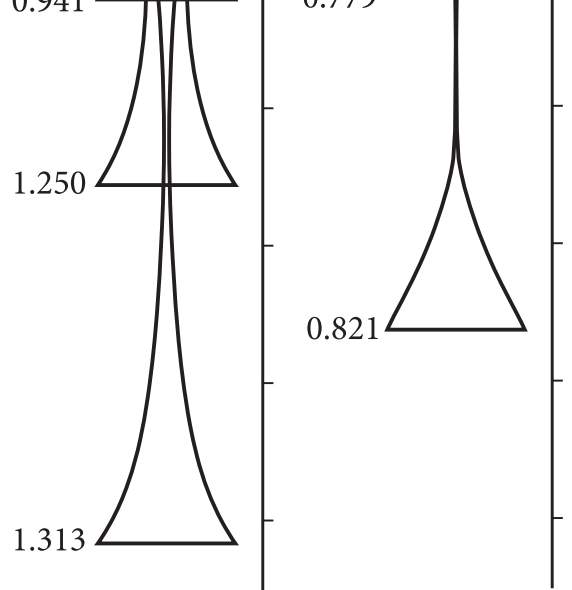

(e) $\mathrm{n}=3$

$\Lambda=5.0$

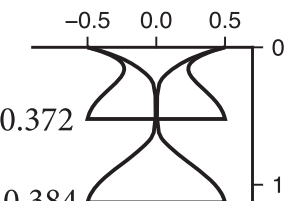

$-2$

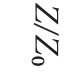

$-3$

4

$-5$

Figure 9. Evolving slab shapes predicted by DRIP with Newtonian $(n=1)$ and composite Newtonian/non-Newtonian $(n=3, \Lambda>0)$ rheology and a constant convergence rate $U_{0}$. (a) Model geometry showing the coordinates used. (b)-(e) Shape of the slab as a function of dimensionless time $t / t_{0}$. The timescale $t_{0}$ is defined by (32), $Z_{0}=U_{0} t_{0}$ and $\Lambda$ is defined by (35). Note that the horizontal scale $\left(=h_{0}\right)$ is different from the vertical scale $\left(=Z_{0}\right)$.

The thickness is a minimum at $\tau=t / 2$, whence

$h_{\min }=h_{0}\left(1-\frac{t^{2}}{4 t_{0}^{2}}\right)$.

The minimum thickness therefore becomes zero at a finite time $t=$ $2 t_{0}$.

Next, we integrate (33) subject to the condition $Z(\tau=t)=0$ to obtain

$Z=\frac{8 \eta_{1}}{g \delta \rho} \frac{1}{\left(4 t_{0}^{2}-t^{2}\right)^{1 / 2}}$

$$
\left\{\tan ^{-1}\left[\frac{t}{\left(4 t_{0}^{2}-t^{2}\right)^{1 / 2}}\right]-\tan ^{-1}\left[\frac{2 \tau-t}{\left(4 t_{0}^{2}-t^{2}\right)^{1 / 2}}\right]\right\}
$$

The total length of the slab is $Z_{\max }=Z(\tau=0)$, or

$Z_{\max }=\frac{16 \eta_{1}}{g \delta \rho} \frac{1}{\left(4 t_{0}^{2}-t^{2}\right)^{1 / 2}} \tan ^{-1}\left[\frac{t}{\left(4 t_{0}^{2}-t^{2}\right)^{1 / 2}}\right]$

Fig. 9(b) shows the shape of the slab at four values of the dimensionless time $t / t_{0}$. The slab thins and lengthens at an accelerating rate. The thinnest cross-section of the slab is always at its midpoint, but corresponds to different material sections at different times. The thickness of the leading end of the slab is always $h_{0}$ because the axial stress there is zero by hypothesis. Even though the minimum thickness decreases to zero in a finite time, the length of the slab becomes infinite at that time.

\subsection{Non-Newtonian slabs}

The governing eqs (33) and (34) cannot be solved analytically for non-Newtonian cases with $n=3$ and $\Lambda>0$, and so numerical methods are necessary. We used a standard fourth-order Runge-Kutta

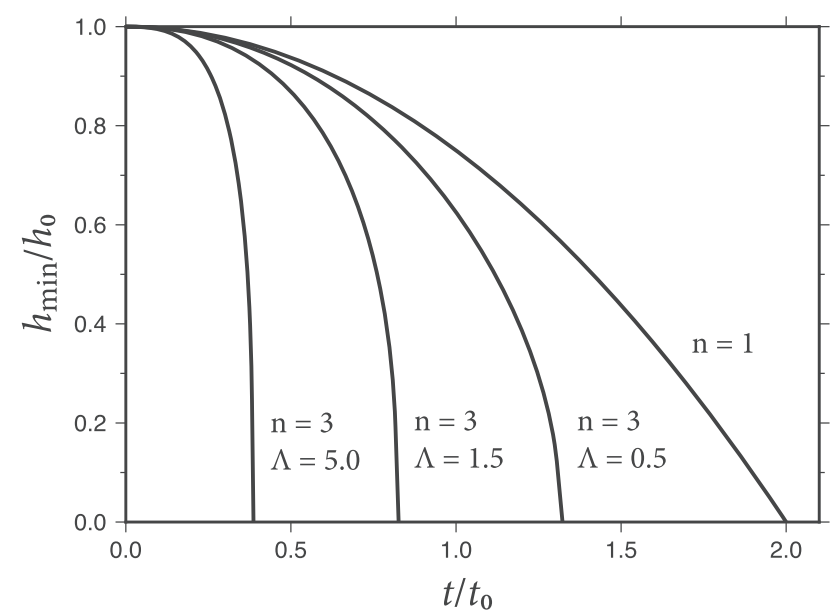

Figure 10. Minimum thickness of the slabs shown in Fig. 9 as functions of time.

algorithm to solve (34), and then solved (33) using a second-order midpoint scheme (the lesser accuracy of the latter is unavoidable because the function $h$ that appears in (33) is only known numerically.) Figs 9(c)-(e) shows the evolving shape of the slab for $\Lambda=0.5,1.5$ and 5.0. The evolution for $\Lambda=0.5$ is qualitatively similar to the Newtonian case $n=1$, but a given slab length is reached earlier and the minimum thickness for that length is less. For $\Lambda=1.5$ and 5.0, the slab quickly reaches a configuration comprising thick upper and lower ends connected by a very thin film. The numerical code was unable to proceed beyond the last configurations in Figs 9(d) and (e) because they correspond to times just before break-off. Fig. 10 shows the minimum thickness of the slab as a function of time for 


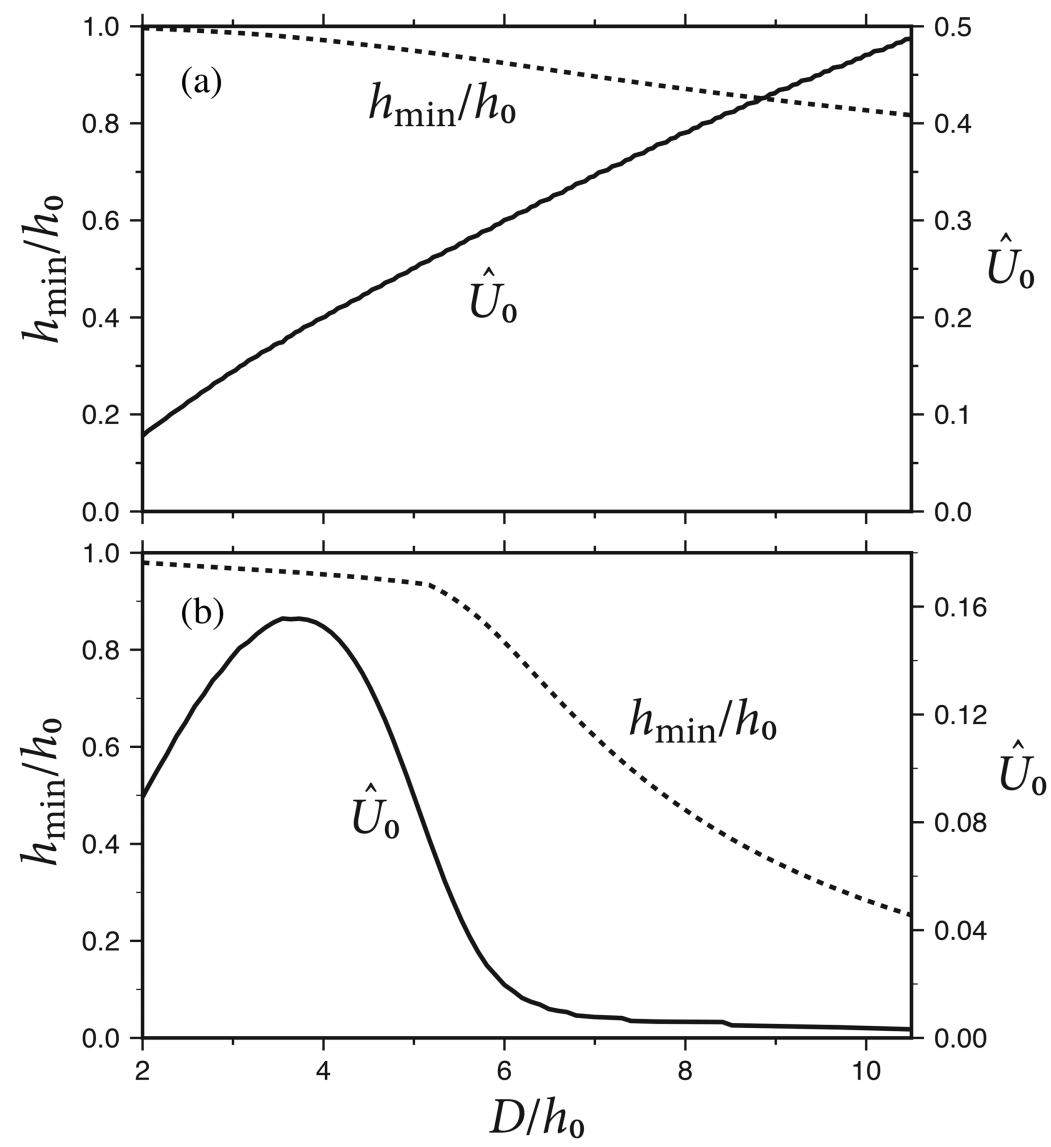

Figure 11. Evolution of the dimensionless convergence rate $\hat{U}_{0} \equiv U_{0} \eta_{0} / g \delta \rho h_{0}^{2}$ and the minimum slab thickness $h_{\min }$ as functions of the depth $D$ of the leading end of the slab, for the case of (a) Figs 6(b) and (b) Fig. 8(a).
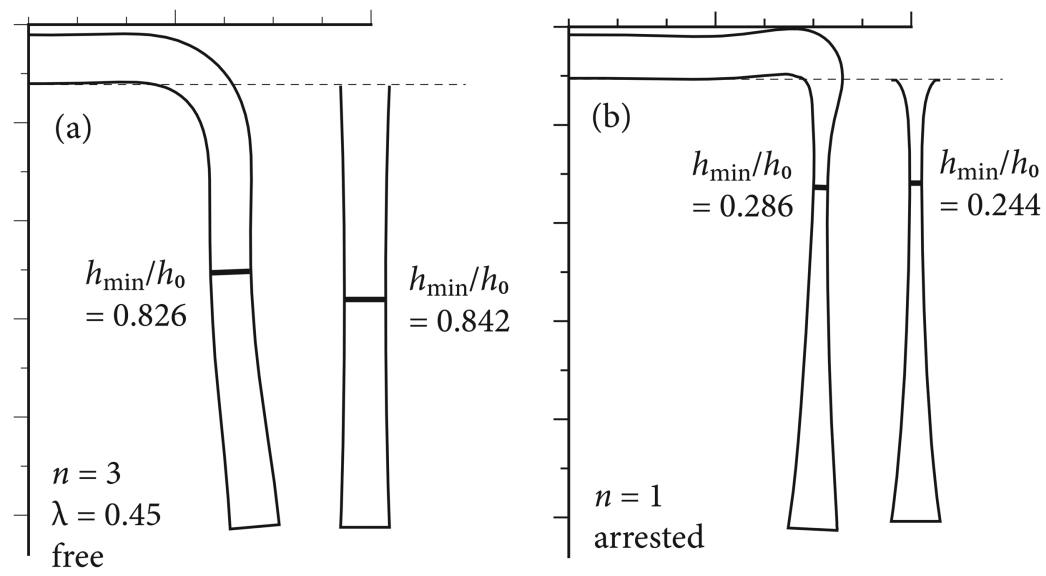

Figure 12. Comparison of the predictions of the 2-D BITS and 1-D DRIP models for a viscosity contrast $\gamma=100$. (a) Free subduction with $n=3$ and $\lambda=0.45$. (b) arrested subduction with $n=1$. The left-hand image in each panel is the shape predicted by BITS, and the right-hand image is the shape predicted by DRIP for the same convergence rate history as in the BITS model. The minimum thickness of each slab is indicated by a horizontal bar. Each image corresponds to the time at which the depth of the slab tip is approximately $10 h_{0}$.

the same four cases as in Figs 9(b)-(e). For the non-Newtonian cases the break-off time is a monotonically decreasing function of $\Lambda$.

\subsection{Variable convergence rate}

The solutions presented above assume that the convergence rate $U_{0}$ is constant, which is never the case for our BITS solutions.
As an illustration, Fig. 11 shows the dimensionless convergence rate $\hat{U}_{0} \equiv U_{0} \eta_{0} / g \delta \rho h_{0}^{2}$ and the minimum slab thickness $h_{\min } / h_{0}$ as functions of the depth $D$ of the leading edge of the slab for the solution shown in (a) Figs 6(a) and (b) Fig. 8(a). The velocity $U_{0}$ was calculated from the BITS solutions as the mid-surface-parallel velocity of the sheet at the depth $\left(=h_{0}\right)$ corresponding to the base of the sheet's horizontal part. 


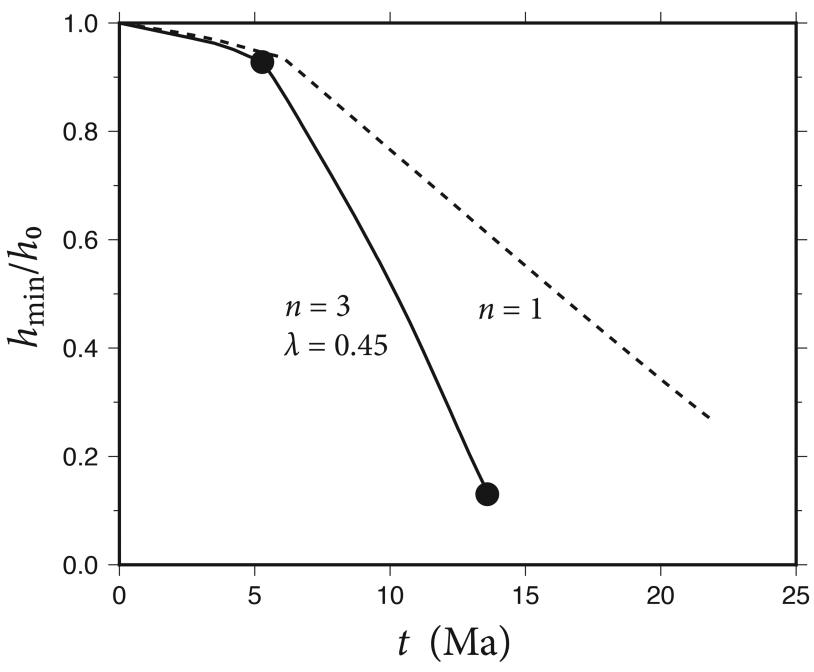

Figure 13. Minimum thickness $h_{\min }$ of the slab as a function of dimensional time, for the arrested subduction cases of Fig. 8. The dimensional time is calculated as $t=\eta_{0} \hat{t} /\left(h_{0} g \delta \rho\right)$ where $\hat{t}$ is the dimensionless (model) time, $h_{0}=100 \mathrm{~km}, \delta \rho=80 \mathrm{~kg} \mathrm{~m}^{-3}$ and $\eta_{0}=5 \times 10^{20} \mathrm{~Pa} \mathrm{~s}$ is an average viscosity of the upper mantle (Mitrovica et al. 2015). The two filled circles are separated by $8.5 \mathrm{Ma}$.

In free subduction (Fig. 11a), the convergence rate increases monotonically due to the continually increasing pull of the lengthening slab. In arrested subduction (Fig. 11b), by contrast, the initial increase of the convergence rate is stopped and then reversed by the arrival at the trench of the positively buoyant part of the plate. The relatively high values of $\hat{U}_{0}$ in Fig. 11(a) and in the initial stages $D / h_{0}<5.2$ of Fig. 11(b) are associated with small amounts of slab thinning $\left(h_{\min } / h_{0}>0.9\right)$. In the arrested case, however, the strong decrease of the convergence rate for $4<D / h_{0}<7$ causes the thinning to accelerate starting at $D / h_{0} \approx 5.2$.

We now compare the predictions of the BITS and DRIP models for two representative cases corresponding to Figs 6(b) and 8(a). The DRIP solutions are obtained using the same numerical algorithm as previously, but using the variable convergence rate $U_{0}(t)$ supplied by the BITS solution for the same values of $n, \gamma$ and $\lambda$ (Fig. 11.)

Fig. 12 shows the shapes of the slab predicted by the BITS and DRIP models for a viscosity ratio $\gamma=100$, for cases in which the subduction is (a) free and (b) arrested. The numerical solutions shown correspond to times at which the depth of the slab tip is $10 h_{0}$. In the free case, the amount of thinning $1-h_{\min } / h_{0}=0.174$ predicted by BITS is reproduced to within 9 percent by the DRIP model. The depth at which the minimum occurs is also reasonably well predicted by DRIP to within an absolute error of $\approx 0.6 h_{0}$. In the arrested case (Fig. 12b), DRIP predicts the amount of thinning to within 6 per cent, and the depth of the minimum to within an error of only $0.1 h_{0}$.

In the foregoing discussions of evolving subduction, the depth of the leading edge of the slab served as a time-like variable that increases monotonically as subduction proceeds. However, it is also useful to know the true time in Ma associated with the different stages of subduction seen in our models. To this end, we show in Fig. 13 the minimum thickness $h_{\min }$ of the slab as a function of time in Ma, for the two arrested subduction cases of Fig. 8. The solid (non-Newtonian) curve is to the left of the dashed (Newtonian) one, indicating that the presence of a shear-thinning component of the rheology leads to more rapid thinning. For the ranges of parameters explored in our models, we find that the main phase of thinning occupies as little as 8.5 Ma.

\section{DISCUSSION}

The work presented here has aimed to achieve a compromise between geophysical realism on the one hand and simplicity and physical clarity on the other. Several features of the model tend towards realism: these include the continuous curvature of the subducting sheet; the sheet's composite Newtonian and non-Newtonian rheology; deformation of the sheet by a combination of stretching and bending; mechanical interaction between the sheet and its surroundings; and the possibility of embedding a buoyant continental plate in the sheet. However, all these diverse features are compatible with a model of remarkable simplicity. That model has the form of a single integral equation, the BITS equation, which governs the instantaneous velocity of points on the mid-surface of a sheet with a given geometry and thickness. It is worth emphasizing that this reduction of the problem to a single equation is possible because the local Rayleigh dissipation potential for our chosen rheology can be evaluated analytically. More generally, the BITS approach can be used with any rheological law that permits analytical evaluation of the dissipation potential.

Because the viscosity in both the BITS and DRIP models is a harmonic mean of Newtonian and non-Newtonian viscosities, each model necessarily involves a dimensionless parameter that measures the importance of the non-Newtonian response relative to the Newtonian one. No fewer than four such parameters were introduced in this work, and the reader may wonder why so many were needed. To summarize, the parameters introduced were the following:

$\lambda_{b}=\frac{\eta_{1}}{B_{n}}(|\dot{K}| h)^{1-1 / n}, \quad \lambda_{s}=\frac{\eta_{1}}{B_{n}}|\Delta|^{1-1 / n}$,

$\lambda=\frac{\eta_{1}}{B_{n}}\left(\frac{h_{0} g \delta \rho}{\eta_{1}}\right)^{1-1 / n}, \quad \Lambda=\frac{\eta_{1}}{B_{n}}\left(\frac{g \delta \rho U_{s}}{4 \eta_{1}}\right)^{(n-1) / 2 n}$.

All of the above parameters have the same physical meaning: they are characteristic values of the ratio $\eta_{1} / \eta_{2}$ of the Newtonian to the non-Newtonian viscosity. Because the sheet deforms predominantly by the mechanism (diffusion or dislocation creep) having the lower viscosity, a larger value of $\eta_{1} / \eta_{2}$ corresponds to a greater dominance of dislocation creep. The differences among the parameters (41) are functions of the kind of deformation assumed. $\lambda_{b}$ is appropriate for an ideal pure bending deformation with an imposed rate of change of curvature $\dot{K}$. Similarly, $\lambda_{s}$ is used for an ideal pure stretching deformation with an imposed stretching rate $\Delta$. In subducting sheets, however, neither $\dot{K}$ nor $\Delta$ is imposed a priori; instead, both are determined dynamically by the buoyancy force acting on a sheet with its given geometry. In that case, it makes sense to use in place of $\dot{K} h$ or $\Delta$ a characteristic buoyancy-driven strain rate $h_{0} g \delta \rho / \eta_{1}$. The result is the parameter $\lambda$. Finally, $\Lambda$ is appropriate for a situation like that envisioned by the DRIP model, in which the sheet is ejected from a slot with a characteristic velocity $U_{s}$. In all four cases the value of the parameter tends to infinity in the limit of a pure dislocation creep (power-law) rheology with no diffusion creep (Newtonian) component.

Next, we remark upon the degree to which necking instabilities are localized in space, that is, in depth. In our 2-D BITS simulations, thinning of the slab is not localized, but is instead spread out over a depth range that is many times the sheet thickness ( $c f$. Figs 6-8). By contrast, the 2-D numerical solutions of Schmalholz (2011) show strong localization for $n=4$; only for $n=1$ (the Newtonian case) is 
the thinning broadly distributed (compare Figs 3 and 4 of Schmalholz (2011)). We think that the difference between our solutions and those of Schmalholz (2011) is due to the different rheologies used. Schmalholz (2011) assumed a pure power-law (shear-thinning) rheology, for which the effective viscosity becomes infinite as the strain rate tends to zero. Such a rheology creates strong localization by dividing the slab into a low-viscosity neck where deformation is rapid, and surrounding zones of very high viscosity where the strain rate is small. This can be clearly seen in fig. 4(d) of Schmalholz (2011), where the ratio of the viscosity in the non-deforming region to that in the neck is at least $10^{3}$. In our solutions, on the other hand, the use of a composite Newtonian/non-Newtonian rheology provides an upper bound on the viscosity, which can never exceed $\eta_{1}$ no matter how small the strain rate may be. The result is that viscosity variations due to differences in strain rate are smaller, and the deformation of the slab less localized, than for a pure power-law rheology.

A related issue concerns the definition of the word 'break-off' itself. Schmalholz (2011) distinguished between 'breaking off' and 'dripping off', the former occurring for non-Newtonian slabs $(n>$ $1)$ and the latter for Newtonian ones $(n=1)$. The distinction is based on the degree of localization of the thinning and implicitly assumes a pure power-law rheology, as discussed in the previous paragraph. We prefer an alternate definition of break-off as a finite-time singularity where the slab's minimum thickness vanishes in a finite time and at a finite depth. The 'finite depth' qualifier is important because DRIP predicts that a stretching Newtonian slab becomes infinitely long at the finite time when its minimum thickness vanishes. According to our strict definition, then, Newtonian slabs cannot experience break-off. The question remains whether slabs with composite Newtonian/non-Newtonian rheology behave differently. Unfortunately no long-time analytical solutions are available for this case, and so we have to rely on indications provided by numerical solutions such as those of Figs 9(c)-(e). Those solutions suggest that the effect of non-Newtonian rheology is to divide the sheet into two slowly deforming ends joined by a thin and rapidly stretching film or 'neck'. It is of course impossible to run a numerical model with finite resolution all the way to break-off, and so we cannot be sure that the length of the neck remains finite at the finite time when its thickness vanishes. If it does, then slabs with composite rheology can experience break-off in the strict sense of the term. But geophysically speaking the point is probably moot, since thermal diffusion and viscous dissipation will cause detachment well before necks as thin as those in Figs 9(d) and (e) can be formed.

Our DRIP model predicts that a Newtonian slab with a constant convergence rate thins quadratically with time [eq. (38) and Fig. 10] whereas Schmalholz (2011) predicts that the thinning of a Newtonian slab is linear with time. The reason for this difference is that DRIP is a 1-D model that predicts how stretching of the slab is distributed at each instant as a function of depth, whereas the model of Schmalholz (2011) is a zero-dimensional one that focuses on the behaviour of a particular material section. Now an important prediction of DRIP is that the thinnest cross-section of the slab is not always the same material section. This conclusion follows directly from the analytical solution (37) for a Newtonian slab, and also from a small-time analytical solution (Appendix E) for a slab with a weakly non-linear composite rheology. If we follow the thinning of a particular material section $\tau=\tau_{0}$ (say) of a Newtonian slab, then (37) shows that the thickness of that section evolves as

$h=h_{0}\left[1+\frac{\tau_{0}\left(\tau_{0}-t\right)}{t_{0}^{2}}\right]$.
Eq. (42) shows that any particular material section thins linearly with time, even though the sheet as a whole thins quadratically with time. The conclusion is that the non-material character of the section that happens to be thinnest at a given instant is crucial in determining the rate of slab thinning. However, in the limiting case of a slab of constant area produced by a convergence rate that diminishes instantaneously to zero, the thinnest section will be the material section at the very top of the slab. The model of Schmalholz (2011) can be expected to apply in this case.

A central conclusion of this work is that the timing and depth of slab break-off are highly sensitive to the convergence rate $U_{0}(t)$. In fact, $U_{0}(t)$ has a dual role that is made clear by the DRIP eqs (25) and (31). In (25), which governs the Eulerian depth coordinate $Z(\tau, t), U_{0}(\tau)$ appears explicitly on the right-hand side. This means that $Z(\tau, t)$, and by implication the depth at which slab break-off occurs, depends on the detailed history of the convergence rate. In the eq. (31) that governs the slab thickness $h(\tau, t)$, by contrast, $U_{0}(t)$ appears only in the integral $F(\tau)=\int_{0}^{\tau} U_{0}(\zeta) \mathrm{d} \zeta$. This implies that the amount of thinning experienced by the slab depends not on the details of the convergence history $U_{0}(t)$, but only on the 'accumulated convergence' $F(\tau)$. Accordingly, we need to examine our BITS solutions with two questions in mind: how the properties of the solutions depend on the accumulated convergence, and how they depend on the detailed history of the convergence rate. We now address these questions in turn.

The influence of accumulated convergence on slab thinning is illustrated by a comparison of our BITS solutions for free and arrested subduction. In the free case, thinning of the slab is limited to $h_{\text {min }} \approx 0.4 h_{0}$ even for large penetration depths $\approx 10 h_{0}$ and for strongly non-Newtonian rheology with $\lambda=4.5$ (see Figs 6 and 7). In arrested subduction, by contrast, the BITS solutions predict much stronger slab thinning than in the free case for the same values of $\gamma$ and $\lambda$ (compare Figs 6 and 8). The reason is that beyond the initial stage of oceanic subduction the accumulated convergence in arrested subduction increases only slowly due to the reduced convergence rate, whereas the accumulated convergence increases relatively rapidly in the free case (compare Figs 11a and b).

Further insight into the role of the accumulated convergence can be obtained from an analytical solution of the DRIP equations that is valid for small times $t \ll t_{0}$ and weakly nonlinear rheology $\Lambda$ $\ll 1$. That solution is determined in Appendix E for a constant convergence rate $U_{0}$ and a power-law index $n=3$. It predicts that the minimum thickness $h_{\min }$ of the slab depends on its length $Z_{\max }$ according to

$$
\begin{aligned}
\frac{h_{\min }}{h_{0}} & =1-\frac{1}{4}\left(\frac{Z_{\max }}{Z_{0}}\right)^{2}-\frac{\Lambda}{8}\left(\frac{Z_{\max }}{Z_{0}}\right)^{8 / 3} \\
& =1-\frac{1}{16} \frac{g \delta \rho}{\eta_{1} U_{0}} Z_{\max }^{2}-\frac{1}{32}\left(\frac{1}{4}\right)^{2 / 3} \frac{(g \delta \rho)^{5 / 3}}{B_{3} \eta_{1}^{2 / 3} U_{0}} Z_{\max }^{8 / 3} .
\end{aligned}
$$

The crucial feature of (43) is that the amount of thinning for a given depth of penetration of the slab is inversely proportional to $U_{0}$. Moreover, since $U_{0}$ is constant the accumulated convergence is directly proportional to it. This explains why slab break-off is so much easier to achieve in arrested subduction (lower accumulated convergence) than in free subduction (higher accumulated convergence). This also makes intuitive sense: when $U_{0}$ is smaller, increments of buoyancy-driven stretching have more time to accumulate before the slab reaches a given depth. The general conclusion that low convergence rates favour break-off is in accord with that of Boutelier \& Cruden (2017) based on analog laboratory experiments. However, these authors suggest a primarily thermal explanation in which low 
(1-2 $\left.\mathrm{cm} \mathrm{yr}^{-1}\right)$ convergence rates before continental collision produce warmer and weaker slabs that break off earlier and at shallower depths. Our work suggests an alternative explanation based on the fluid mechanics of viscous dripping.

Turning now to the influence of the convergence history, we note first that our BITS solutions for free subduction predict that the depth of maximum thinning is about midway between the top and bottom of the slab (Figs 6 and 7). In arrested subduction, however, the depth of maximum thinning is pushed up to shallower depths, 15-25 percent of the way from the top to the bottom of the slab (Fig. 8). This shallowing of slab break-off can be intuitively understood if one considers the extreme limit of arrested subduction: a convergence rate that is very large and constant for a certain time and then instantaneously falls to zero. This is equivalent to putting in place a slab of a certain length with nearly constant thickness, and then letting it stretch under its own weight as it 'hangs from the ceiling' with no more material supplied from above. In this situation thinning will be concentrated in the very uppermost part of the slab due to the effectively clamped boundary condition at the top. The real situation is of course less extreme, with a convergence rate that decreases continuously after the initial stage of oceanic subduction (Fig. 11b), but the general conclusion that a decreasing convergence rate pushes the break-off point to shallower depths remains valid. This conclusion is consistent with the prediction of the 'slowdown subduction' model of $\mathrm{Li}$ et al. (2002).

A natural question to ask at this point is whether one could infer effective values of the rheological parameters $\gamma, \lambda$ and $n$ from seismological observations of the existence and depth of slab break-off in particular subduction zones. We generally believe that it is important to attempt to constrain model parameters, and in that light our Appendix B estimates a range of values of $\lambda$ based on experimental data for olivine. However, there are several reasons why it would be difficult to use seismological data for the same purpose. First, our numerical solutions require an intact (even if thinned) slab, and cannot model the post-break-off stage that is revealed by tomographic images and/or earthquake hypocentre distributions. Second, the convergence histories at subduction zones where slabs appear to have broken off are generally rather poorly known, which makes it difficult to run realistic BITS simulations. Third, our assumed composite rheology, while more realistic than that used in a number of previous studies, is still far too idealized to represent the lithosphere. This point is discussed further below where we summarize some of the unrealistic aspects of our model. And finally, there is a fundamental conundrum connected with slab break-off that occurs during free oceanic subduction, which seems to require unrealistically high values of $\lambda$. Geophysical examples are the central Sunda arc of Indonesia, where Kundu \& Gahalaut (2011) used earthquake hypocentres to identify a horizontal slab tear in the Indo-Australian slab at 300-500 km depth; and northern Central America, where P-wave tomographic images reveal a detached slab (Rogers et al. 2002). The current convergence rates in both regions are quite high [67 and 73-85 $\mathrm{mm} \mathrm{yr}^{-1}$, respectively; DeMets et al. (2010)], and so there is little hope of explaining the break-off by invoking low values of accumulated convergence. The only other possibility seems to be a very large value of $\lambda$, much greater than the value 4.5 that failed to yield break-off in our free subduction simulations (see Figs 6 and 7). But then the question arises: if $\lambda$ is so large, why does slab break-off not occur in all subduction zones on Earth? One possibility is that the data in Sunda and Central America reveal not a completely detached slab, but rather a substantially thinned but still intact one, somewhat like Fig. 6(c) but with a still greater value of $\lambda$. Another possibility is that 3-D effects such as propagation of slab windows and tears (van Hunen \& Allen 2011; von Tscharner et al. 2014) may cause faster slab break-off than is predicted by our 2-D models. Clearly more work is needed to achieve a fully satisfactory agreement between geophysical observations and modelling.

We close by summarizing a few unrealistic aspects of our models that readers should keep in mind.

First, our models are 2-D (BITS) or 1-D (DRIP), and neglect variations along the strike of the subducting slab. As already mentioned, a potentially important 3-D effect is the lateral propagation of slab 'tears' and 'windows', which may occur more rapidly than necking that is uniform along strike. Indeed, numerical and analog studies of tear propagation tend to suggest timescales of no more than a few Ma for the process (Yoshioka \& Wortel 1995; van Hunen \& Allen 2011; Boutelier \& Cruden 2017).

Secondly, our models are isothermal, and therefore ignore the potentially important effects of thermal diffusion and viscous dissipation of energy, both of which will tend progressively to warm the slab as it descends in the mantle. As a result the resistance of the slab to stretching and bending should decrease with downdip distance along the slab. This will enhance necking instabilities and lead to shorter break-off times than those typical of our models ( $c f$. Fig. 13).

Finally, in our composite viscosity law (11), we have assumed that the Newtonian viscosity $\eta_{1}$ and the power-law stiffness $B_{n}$ are constants that do not vary across the sheet. A consequence of this assumption is that the viscosity of our model sheets is symmetric about the mid-surface $z=0$. However, this is obviously not the case in the Earth, because the temperature varies across the lithosphere by some $1300 \mathrm{~K}$. Lithospheric rheology is therefore highly asymmetric, varying from brittle at the top to viscous at the bottom. We are currently working to take this feature into account using a modified version of the thin-sheet theory used in this study.

\section{A CKNOWLEDGEMENTS}

We are grateful to S. Schmalholz, Z. Li and T. Duretz for careful reviews that helped greatly to improve the manuscript. This work was supported by funds provided by the CNRS and the Universite Paris-Sud to the FAST laboratory.

\section{REFERENCES}

Andrews, E.R. \& Billen, M.I., 2009. Rheologic controls on the dynamics of slab detachment, Tectonophys., 464, 60-69.

Baumann, C., Gerya, T.V. \& Connolly, J.A.D., 2010. Numerical modelling of spontaneous slab breakoff dynamics during continental collision, Geol. Soc. Lond. Spec. Publ., 332, 99-114.

Bellas, A., Zhong, S., Bercovici, D. \& Mulyukova, E., 2018. Dynamic weakening with grain-damage and implications for slab detachment, Phys. Earth planet. Int., 285, 76-90.

Boutelier, D. \& Cruden, A.R., 2017. Slab breakoff: insights from 3D thermomechanical analogue modelling experiments, Tectonophys., 694, 197213.

Broerse, T., Norder, B., Govers, R., Sokoutis, D., Willingshofer, E. \& Picken, S.J., 2019. New analogue materials for nonlinear lithosphere rheology, with an application to slab break-off, Tectonophys., 756, 73-96.

Buiter, S.J.H., Govers, R. \& Wortel, M.J.R., 2002. Two-dimensional simulations of surface deformation caused by slab detachment, Tectonophys., 354, 195-210.

Burkett, E.R. \& Billen, M.I., 2010. Three-dimensionality of slab detachment due to ridge-trench collision: laterally simultaneous boudinage versus tear propagation, Geochem. Geophys. Geosyst., 11, Q11012. 
Chatelain, J.-L., Molnar, P., Prévot, R. \& Isacks, B., 1992. Detachment of part of the downgoing slab and uplift of the New Hebrides (Vanuatu) islands, Geophys. Res. Lett., 19, 1507-1510.

Cortez, R., 2002. The method of regularized Stokeslets, SIAM J. Sci. Comput., 23, 1204-1225.

Davies, J.H. \& von Blanckenburg, F., 1995. Slab breakoff: a model of lithosphere detachment and its test in the magmatism and deformation of collisional orogens, Earth planet. Sci. Lett., 129, 85-102.

DeMets, C., Gordon, R.G. \& Argus, D.F., 2010. Geologically current plate motions, Geophys. J. Int., 181, 1-80.

Duretz, T., Schmalholz, S.M. \& Gerya, T.V., 2012. Dynamics of slab detachment, Geochem. Geophys. Geosyst., 13, Q03020.

Faccenna, C., Bellier, O., Martinod, J., Piromallo, C. \& Regard, V., 2006. Slab detachment beneath eastern Anatolia: a possible cause for the formation of the North Anatolian fault, Earth planet. Sci. Lett., 242, 85-97.

Gerya, T.V., Yuen, D.A. \& Maresch, W.V., 2004. Thermomechanical modelling of slab detachment, Earth planet. Sci. Lett., 226, 101-116.

Isacks, B. \& Molnar, P., 1969. Mantle earthquake mechanisms and the sinking of the lithosphere, Nature, 223, 1121-1124.

Karato, S.-I. \& Wu, P., 1993. Rheology of the upper mantle: a synthesis, Science, 260, 771-778.

Kundu, B. \& Gahalaut, V.K., 2011. Slab detachment of subducted IndoAustralian plate beneath Sunda arc, Indonesia, J. Earth Syst. Sci., 120, 193-204.

Larsen, T.B., Yuen, D.A. \& Malevsky, A.V., 1995. Dynamics consequences on fast subducting slabs from a self-regulating mechanism due to viscous heating in variable viscosity convection, Geophys. Res. Lett., 22, 12771280 .

Li, L., Liao, X. \& Fu, R., 2002. Slab breakoff depth: a slowdown subduction model, Geophys. Res. Lett., 29, 1041.

McKenzie, D.P., 1969. Speculations on the consequences and causes of plate motions, Geophys. J. R. Astr. Soc., 18, 1-32.

Mitrovica, J.X., Hay, C.C., Morrow, E., Kopp, R.E., Dumberry, M. \& Stanley, S., 2015. Reconciling past changes in earth's rotation with 20th century global sea-level rise: resolving Munk's enigma, Sci. Adv., 1, e1500679.

Pascal, G., Dubois, J., Barazangi, M., Isacks, B.L. \& Oliver, J., 1973. Seismic velocity anomalies beneath the New Hebrides island arc: evidence for a detached slab in the upper mantle, J. geophys. Res., 78, 6998-7004.

Press, W.H., Teukolsky, S.A., Vetterling, W.T. \& Flannery, B.P., 1992. Numerical Recipes in Fortran 77, 2nd edn, Cambridge Univ. Press.
Regard, V., Faccenna, C., Bellier, O. \& Martinod, J., 2008. Laboratory experiments of slab break-off and slab dip reversal: insight into the Alpine Oligocene reorganization, Terra Nova, 20, 267-273.

Ribe, N.M., 2010. Bending mechanics and mode selection in free subduction: a thin-sheet analysis, Geophys. J. Int., 180, 559-576.

Rogers, R.D., Karason, H. \& van der Hilst, R.D., 2002. Epeirogenic uplift above a detached slab in northern Central America, J. Geol. Soc., 30, 1031-1034.

Schmalholz, S.M., 2011. A simple analytical solution for slab detachment, Earth planet. Sci. Lett., 304, 45-54.

Ton, S.Y.M.W.A. \& Wortel, M.R.R., 1997. Slab detachment in continental collision zones: an analysis of controlling parameters, Geophys. Res. Lett., 24, 2095-2098.

van Hunen, J. \& Allen, M.B., 2011. Continental collision and slab break-off: a comparison of 3-D numerical models with observations, Earth planet Sci. Lett., 302, 27-37.

von Blanckenburg, F. \& Davies, J.H., 1995. Slab breakoff: a model for syncollisional magmatism and tectonics in the Alps, Tectonics, 14, 120131.

von Tscharner, M., Schmalholz, S.M. \& Duretz, T., 2014. Three-dimensional necking during viscous slab detachment, Geophys. Res. Lett., 41, 4194 4200 .

Westaway, R., 1993. Quaternary uplift of southern Italy, J. geophys. Res., 98, 21 741-21 772 .

Wilson, S.D.R., 1988. The slow dripping of a viscous fluid, J. Fluid Mech., 190, 561-570.

Wortel, M.J.R. \& Spakman, W., 1992. Structure and dynamics of subducted lithosphere in the Mediterranean region, Proc. Kon. Ned. Akad. Wetensch., 95, 325-347.

$\mathrm{Xu}$, B. \& Ribe, N.M., 2016. A hybrid boundary-integral/thin-sheet equation for subduction modelling, Geophys. J. R. astr. Soc., 206, 1552-1562.

Yoshioka, S. \& Wortel, M.J.R., 1995. Three-dimensional numerical modeling of detachment of subducted lithosphere, J. geophys. Res., 100, $20223-$ 20244.

Zor, E., 2011. Tomographic evidence of slab detachment beneath eastern Turkey and the Caucasus, Geophys. J. Int., 175, $1273-1282$.

\section{APPENDIX A: EVALUATION OF THE RAYLEIGH Dissipation POTENTIAL}

Starting from the expression (13) for the fibre stress, we write

$\sigma_{s s}^{ \pm}=4 \Gamma\left(\frac{1}{\eta_{1}}+\frac{1}{B_{n}( \pm \Gamma)^{1 / n-1}}\right)^{-1}, \quad \Gamma=\Delta-\dot{K} z$,

where the $+\operatorname{sign}$ is for $\Gamma>0$ and the - sign for $\Gamma<0$. Next, define local RDPs

$\phi^{ \pm}=\frac{1}{2} \int_{-h / 2}^{h / 2} e_{s s} \sigma_{s s}^{ \pm} \mathrm{d} z=2 \int_{-h / 2}^{h / 2} \Gamma^{2}\left(\frac{1}{\eta_{1}}+\frac{1}{B_{n}( \pm \Gamma)^{1 / n-1}}\right)^{-1} \mathrm{~d} z$.

At this point it is helpful to define dimensionless (hatted) variables

$z=h_{0} \hat{z}, \quad h=h_{0} \hat{h}, \quad \Delta=\frac{h_{0} g \delta \rho}{\eta_{0}} \hat{\Delta}, \quad \dot{K}=\frac{g \delta \rho}{\eta_{0}} \hat{K}, \quad \phi=\frac{h_{0}^{3} g^{2} \delta \rho^{2}}{\eta_{0}} \hat{\phi}$.

The dimensionless local RDP is then

$\hat{\phi}^{ \pm}=\gamma \int_{-\hat{h} / 2}^{\hat{h} / 2} \frac{2(\hat{\Delta}-\hat{\dot{K}} \hat{z})^{2}}{1+\beta[ \pm(\hat{\Delta}-\hat{\dot{K}} \hat{z})]^{1-1 / n}} \mathrm{~d} \hat{z}$,

where $\beta=\gamma^{1-1 / n} \lambda$.

Now define the dimensionless indefinite integrals

$A_{ \pm}(\hat{z})=\int \frac{2(\hat{\Delta}-\hat{\dot{K}} \hat{z})^{2}}{1+\beta[ \pm(\hat{\Delta}-\hat{\dot{K}} \hat{z})]^{1-1 / n}} \mathrm{~d} \hat{z}$. 
The integrals $A_{ \pm}$can only be evaluated analytically if a particular value of $n$ is chosen. As an example, consider $n=3$, a value close to that for olivine deforming by dislocation creep. We then find

$A_{ \pm}=\hat{\dot{K}}^{-1}\left\{ \pm \frac{6( \pm \hat{\Gamma})^{1 / 3}}{\beta^{4}}-\frac{2 \hat{\Gamma}}{\beta^{3}} \pm \frac{6( \pm \hat{\Gamma})^{5 / 3}}{5 \beta^{2}} \mp \frac{6( \pm \hat{\Gamma})^{7 / 3}}{7 \beta} \mp \frac{6 \tan ^{-1}\left[\beta^{1 / 2}( \pm \hat{\Gamma})^{1 / 3}\right]}{\beta^{9 / 2}}\right\}$,

where $\hat{\Gamma}=\hat{\Delta}-\hat{\dot{K}} \hat{z}$.

To evaluate $\hat{\phi}$ we need to consider four cases: $\hat{\Gamma}>0$ over the whole interval $\hat{z} \in[-\hat{h} / 2, \hat{h} / 2]$ (stretching-dominated deformation); $\hat{\Gamma}<0$ over the whole interval (compression-dominated); $\hat{\Gamma}$ changes sign on the interval with $\hat{\Gamma}(\hat{z}=-\hat{h} / 2)<0$ (bending-dominated with $\dot{K}<0$ ); and $\hat{\Gamma}$ changes sign with $\Gamma(\hat{z}=-\hat{h} / 2)>0$ (bending-dominated with $\dot{K}>0)$. The results for all cases can be summarized as

$\hat{\phi}=\gamma\left[A_{q_{+}}(\hat{h} / 2)-A_{q_{-}}(-\hat{h} / 2)\right]$,

where $q_{ \pm}$are the signs of $\hat{\Gamma}$ at $\hat{z}= \pm \hat{h} / 2$.

\section{APPENDIX B: ESTIMATION OF DIMENSIONLESS RHEOLOGICAL PARAMETERS}

Since the dimensionless group $\lambda$ defined by (15) involves rheological parameters of the sheet alone, its value can be estimated from laboratory data. Here we use the data of table 1 of Karato \& Wu (1993) for olivine deforming by either diffusion creep or dislocation creep. The creep law for olivine can be written as

$\dot{\epsilon}=A_{i}\left(\frac{\sigma}{\mu}\right)^{n_{i}}\left(\frac{b}{d}\right)^{m_{i}} \mathcal{E}_{i}, \quad \mathcal{E}_{i}=\exp \left(-\frac{E_{i}+P V_{i}}{R T}\right)$,

where the subscript $i=1$ is for diffusion creep and $i=2$ is for dislocation creep. In (B1), $\dot{\epsilon}$ is the strain rate, $\sigma$ is the stress, $T$ is the temperature, $P$ is the pressure, $A_{i}$ is a pre-exponential factor, $\mu=80 \mathrm{GPa}$ is the shear modulus, $b=0.5 \mathrm{~nm}$ is the length of the Burgers vector, $d$ is the grain size, $n_{i}$ is the stress exponent, $m_{i}$ is the grain size exponent, $E_{i}$ is the activation energy, $V_{i}$ is the activation volume and $R$ is the gas constant. Eliminating the stress $\sigma$ using the relation $\sigma=2 \eta_{i} \dot{\epsilon}$, we find

$\eta_{1}=\frac{\mu}{2}\left(\frac{d}{b}\right)^{m_{1}}\left(A_{1} \mathcal{E}_{1}\right)^{-1}, \quad B_{n_{2}}=\frac{\mu}{2}\left(A_{2} \mathcal{E}_{2}\right)^{-1 / n_{2}}$

where the relations $m_{2}=0$ and $n_{1}=1$ have been used. To estimate $\lambda$, we choose $\mathrm{P}=6.6 \mathrm{GPa}, d=0.001 \mathrm{~m}, g=9.81 \mathrm{~m} \mathrm{~s}{ }^{-2}, h_{0}=80 \mathrm{~km}$ and $\delta \rho=80 \mathrm{~kg} \mathrm{~m}^{-3}$. We also use the values of $A_{i}, E_{i}, V_{i}, n_{2}$ and $m_{1}$ from the tabulation of Karato \& Wu (1993) for dry olivine. It remains to specify the temperature $T$, a choice that strongly influences the estimate of $\lambda$. Consider first $T=1000 \mathrm{~K}$, a temperature roughly midway between the surface temperature $(273 \mathrm{~K})$ and the temperature of the ambient mantle (potential temperature $\approx 1625 \mathrm{~K})$. Evaluating $(\mathrm{B} 2)$ and substituting the results into (15), we find $\lambda=0.26$. However, we also find $\eta_{1}=1.5 \times 10^{28} \mathrm{~Pa}$, which is probably much higher than the true maximum viscosity inside a slab. This leads us to make another estimate of $\lambda$ by choosing $T$ such that the viscosity at that temperature is equal to $\gamma$ times a reasonable estimate of the viscosity of the ambient upper mantle, $\eta_{0}=5 \times 10^{20} \mathrm{~Pa} \mathrm{~s}$ (Mitrovica et al. 2015). That temperature is $1445 \mathrm{~K}$ for $\gamma=100$ and $1390 \mathrm{~K}$ for $\gamma=316$. The corresponding values of $\lambda$ are 8.9 for $\gamma=100$ and 6.5 for $\gamma=316$. Given that the best choice of $\lambda$ is highly uncertain, we use a range of intermediate values $0.45<\lambda<4.5$ in our numerical simulations.

Turning to $\lambda_{b}$, we see from the definitions of this parameter and of $\lambda$ that $\lambda_{b} / \lambda=(\gamma \hat{\dot{K}})^{1-1 / n_{2}}$ where $\hat{\dot{K}}=\eta_{0} \dot{K} / g \delta \rho$. Now when $\gamma$ increases, $\hat{\dot{K}}$ decreases because a stiffer slab bends more slowly. Thus it make sense to take $\lambda_{b} / \lambda$ to be independent of $\gamma$ to lowest order. For $\gamma=100$, our numerical simulations for free subduction show that $\hat{\dot{K}}_{\max } \approx 0.12-2.4$, which implies $\lambda_{b} / \lambda \approx 5.9-9.7$. The results for the parameter $\lambda_{s}$ are similar.

When interpreting the estimates just derived, it should not be forgotten that we had to specify the pressure and the grain size in addition to the temperature. The pressure effect on $\lambda$ is not large, because the viscosities of diffusion creep and dislocation creep depend on pressure in roughly similar ways. By contrast, the grain size affects only diffusion creep, and its effect is substantial: increasing it by a factor of 2 increases the viscosity $\eta_{1}$ by $\approx 2^{2.5}=5.7$. Moreover, if grain size depends on stress the standard linear diffusion creep law can become effectively non-linear. The additional uncertainties involved here are partly taken into account by our decision to consider a range of values of $\lambda$ spanning an order of magnitude $(0.45-4.5)$.

\section{APPENDIX C: DISCRETE EQUATIONS FOR A NON-NEWTONIAN SHEET}

We begin by writing down the governing equations in the smooth (continuous) setting. The Lagrangian form of the BITS equation is given by eq. (A20) of Xu \& Ribe (2016) as

$\mathbf{U}(S)=\frac{\gamma-1}{\gamma} \int_{0}^{L_{0}} \mathbf{P}(R) \cdot \mathbf{J}(R, S) \mathrm{d} R-\int_{0}^{L_{0}} l(R) h(R) \mathbf{e}_{2} \cdot \mathbf{J}(R, S) \mathrm{d} R$,

where the sign of the last term has been changed because $\mathbf{e}_{2}$ points upward. The variables $R$ and $S$ are the initial arclength coordinates of material points on the mid-surface. The viscous force vector $\mathbf{P}$ is given by

$\mathbf{P}=-\frac{\partial \mathcal{D}}{\partial \mathbf{U}}$, 


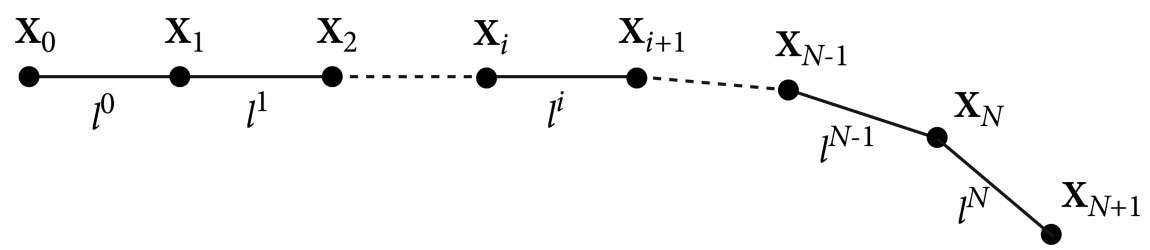

Figure A1. Discrete representation of the sheet's mid-surface, comprising $N+2$ nodes and $N+1$ edges. The Cartesian coordinates of the $i$ th node are $\mathbf{X}_{i}$, and the length of the $i$ th edge is $l^{i}$.

where $\mathcal{D}$ is the global (integrated along the sheet's mid-surface) RDP

$\mathcal{D}=\int_{0}^{L_{0}} \phi(\dot{K}, \Delta, h) \ell \mathrm{d} S$,

where $\ell=\partial s / \partial S$.

We now discretize the foregoing equations. The first step is to define a discrete representation of the sheet's mid-surface as a piecewise continuous curve comprising $N+2$ nodes and $N+1$ edges (fig. A1). The context will prevent confusion of the number $N$ with the fibre stress resultant $N$ defined by (2). Let the Cartesian coordinates of the $i$ th node be $\mathbf{X}_{i}$. Here and henceforth, Latin subscripts are attached to quantities defined on nodes and Latin superscripts to quantities defined on edges. We also introduce Greek indices to denote components of vectors and tensors, and use the Einstein summation convention for these indices only. Thus the Cartesian coordinates of the $i$ th node are $X_{i \alpha}$, where $\alpha=1$ or 2 .

Given the nodal coordinates, we define the length of the edges as $l^{i}=\left|\mathbf{X}_{i+1}-\mathbf{X}_{i}\right|$. The unit tangent vectors along the edges are then

$t^{i \alpha}=\frac{X_{i+1 \alpha}-X_{i \alpha}}{l^{i}}$.

Finally, a superposed tilde indicates the average of a quantity on the two edges adjacent to a given node. Thus e.g.

$\tilde{l}_{i}=\frac{l^{i-1}+l^{i}}{2} \quad(1 \leq i \leq N)$.

In the stiff-sheet limit $\gamma \gg 1$ the discrete representation of (C1) is (Xu \& Ribe 2016)

$U_{i \beta}=\sum_{j=0}^{N+1} P_{j \alpha} J_{i j \alpha \beta}-\sum_{j=0}^{N+1} \tilde{A}_{j} J_{i j 2 \beta}$,

where $A_{j}=(\tilde{l})_{j}$. Defining the quantity $P_{j \alpha}$ to be the vector component $\alpha$ of the force at node $j$, we have

$P_{j \alpha}=-\frac{\partial \mathcal{D}}{\partial U_{j \alpha}}$.

The discretized form of (C3) that reduces to the discretization of Xu \& Ribe (2016) for a Newtonian sheet is

$\mathcal{D}=\sum_{k=0}^{N} \frac{l^{k}}{2}\left\{\phi\left(e_{k}^{b}, e_{s}^{k}, h^{k}\right)+\phi\left(e_{k+1}^{b}, e_{s}^{k}, h^{k}\right)\right\}$

The expression (C8) is obtained by making the substitutions $\dot{K} \rightarrow e_{k}^{b}$ and $\Delta \rightarrow e_{s}^{k}$ and using the trapezoidal rule to approximate the integral (C3). The discrete bending strain rate is

$e_{k}^{b}=\frac{\omega^{k}-\omega^{k-1}}{\tilde{l}_{k}}=\frac{\epsilon_{3 \alpha \beta}}{\tilde{l}_{k}}\left[t^{k \alpha} \frac{U_{k+1 \beta}-U_{k \beta}}{l^{k}}-t^{k-1 \alpha} \frac{U_{k \beta}-U_{k-1 \beta}}{l^{k-1}}\right]$,

where $\omega^{k}$ is the discrete angular velocity of edge $k$. The discrete stretching strain rate is

$e_{s}^{k}=t^{k \alpha} \frac{\left(U_{k+1 \alpha}-U_{k \alpha}\right)}{l^{k}}$.

Substituting (C8) into (C7) and using (C9) and (C10), we obtain

$$
\begin{aligned}
P_{j \alpha}= & -\frac{1}{2} \sum_{k=0}^{N} l^{k}\left\{\mathcal{A}_{\alpha}^{k j}\left(\frac{\partial \phi}{\partial \dot{K}}\right)_{k k}+\mathcal{A}_{\alpha}^{k+1 j}\left(\frac{\partial \phi}{\partial \dot{K}}\right)_{k+1 k}\right. \\
& \left.+\mathcal{B}_{\alpha}^{k j}\left[\left(\frac{\partial \phi}{\partial \Delta}\right)_{k k}+\left(\frac{\partial \phi}{\partial \Delta}\right)_{k+1 k}\right]\right\},
\end{aligned}
$$

where

$\mathcal{A}_{\alpha}^{k j} \equiv \frac{\partial e_{k}^{b}}{\partial U_{j \alpha}}=\frac{\epsilon_{3 \lambda \alpha}}{\tilde{l}_{k}}\left[t^{k \lambda} \frac{\delta_{j k+1}-\delta_{j k}}{l^{k}}-t^{k-1 \lambda} \frac{\delta_{j k}-\delta_{j k-1}}{l^{k-1}}\right]$ 
and

$\mathcal{B}_{\alpha}^{k j} \equiv \frac{\partial e_{s}^{k}}{\partial U_{j \alpha}}=t^{k \alpha} \frac{\left(\delta_{j k+1}-\delta_{j k}\right)}{l^{k}}$.

In (C11),

$$
\left(\frac{\partial \phi}{\partial \dot{K}}\right)_{m k}=\frac{\partial \phi}{\partial \dot{K}}\left(e_{m}^{b}, e_{s}^{k}, h^{k}\right), \quad\left(\frac{\partial \phi}{\partial \Delta}\right)_{m k}=\frac{\partial \phi}{\partial \Delta}\left(e_{m}^{b}, e_{s}^{k}, h^{k}\right) .
$$

Finally, $(\mathrm{C} 11)$ is simplified by neglecting the terms proportional to $(\partial \phi / \partial \dot{K})_{00}$ and $(\partial \phi / \partial \dot{K})_{N+1 N}$ which are small because the bending strains $e_{0}^{b}$ and $e_{N+1}^{b}$ vanish at both ends of the sheet. We thereby obtain

$$
\begin{aligned}
P_{j \alpha}= & -\frac{1}{2}\left\{l^{0} \mathcal{A}_{\alpha}^{1 j}\left(\frac{\partial \phi}{\partial \dot{K}}\right)_{10}+l^{n} \mathcal{A}_{\alpha}^{N j}\left(\frac{\partial \phi}{\partial \dot{K}}\right)_{N N}\right\} \\
& -\frac{1}{2} \sum_{k=1}^{N-1} l^{k}\left\{\mathcal{A}_{\alpha}^{k j}\left(\frac{\partial \phi}{\partial \dot{K}}\right)_{k k}+\mathcal{A}_{\alpha}^{k+1 j}\left(\frac{\partial \phi}{\partial \dot{K}}\right)_{k+1 k}\right. \\
& \left.+\mathcal{B}_{\alpha}^{k j}\left[\left(\frac{\partial \phi}{\partial \Delta}\right)_{k k}+\left(\frac{\partial \phi}{\partial \Delta}\right)_{k+1 k}\right]\right\}
\end{aligned}
$$

Eq. (C6) together with (C15) constitutes a nonlinear system of simultaneous equations that we solved using a globally convergent Newton's method (Press et al. 1992).

\section{APPENDIX D: DISCRETE EQUATIONS FOR A NEWTONIAN SHEET}

The equations of Appendix C take simpler forms if the sheet has a Newtonian rheology. The local Rayleigh dissipation potential is then a sum of contributions due to bending and stretching:

$\phi(\dot{K}, \Delta, h)=\frac{1}{2} \frac{\gamma h^{3}}{3} \dot{K}^{2}+\frac{1}{2} 4 \gamma h \Delta^{2}$.

Now substitute (D1) into (C11) and assume that the bending moment at both ends of the sheet is zero $\left(e_{0}^{b}=e_{N+1}^{b}=0\right)$. The result is

$P_{j \alpha}=-\gamma \sum_{k=0}^{N}\left(\psi_{k} \mathcal{A}_{\alpha}^{k j} e_{k}^{b}+\chi^{k} \mathcal{B}_{\alpha}^{k j} e_{s}^{k}\right)$

where

$\psi_{k}=\frac{1}{6 \tilde{l}_{k}}\left[l^{k-1}\left(h^{k-1}\right)^{3}+l^{k}\left(h^{k}\right)^{3}\right] \quad(k \neq 0), \quad \psi_{0}=0, \quad \chi^{k}=4 h^{k}$.

In view of the definitions (C9) and (C10), (D2) can be written as

$P_{j \alpha}=-\gamma \sum_{k=0}^{N}\left(\mathcal{E}_{\alpha \lambda}^{k j} U_{k+1 \lambda}+\mathcal{F}_{\alpha \lambda}^{k j} U_{k \lambda}+\mathcal{G}_{\alpha \lambda}^{k j} U_{k-1 \lambda}\right)$,

where

$\mathcal{E}_{\alpha \lambda}^{k j}=\psi_{k} \mathcal{A}_{\alpha}^{k j} \epsilon_{3 \gamma \lambda} \frac{t^{k \gamma}}{l_{k}}+\chi^{k} \mathcal{B}_{\alpha}^{k j} t^{k \lambda}$,

$\mathcal{F}_{\alpha \lambda}^{k j}=\psi_{k} \mathcal{A}_{\alpha}^{k j} \epsilon_{3 \gamma \lambda}\left(-\frac{t^{k \gamma}}{l^{k}}-\frac{t^{k-1 \gamma}}{l^{k-1}}\right)-\chi^{k} \mathcal{B}_{\alpha}^{k j} t^{k \lambda}$,

$\mathcal{G}_{\alpha \lambda}^{k j}=\psi_{k} \mathcal{A}_{\alpha}^{k j} \epsilon_{3 \gamma \lambda} \frac{t^{k-1 \gamma}}{l^{k-1}}$.

The discretized BITS equation in the stiff-sheet limit now takes the form

$U_{i \beta}+\gamma \sum_{j=0}^{N+1} \sum_{k=0}^{N}\left(\mathcal{E}_{\alpha \lambda}^{k j} U_{k+1 \lambda}+\mathcal{F}_{\alpha \lambda}^{k j} U_{k \lambda}+\mathcal{G}_{\alpha \lambda}^{k j} U_{k-1 \lambda}\right) J_{i j \alpha \beta}=-\sum_{j=0}^{N+1} \tilde{A}_{j} J_{i j 2 \beta}$.

\section{APPENDIX E: SMALL-TIME ANALYTICAL SOLUTION OF THE DRIP EQUATIONS}

In this appendix, we determine an analytical solution of the DRIP eqs (33), (34) that is valid in the limit of small time $t \ll t_{0}$ and weak rheological non-linearity $\Lambda \ll 1$, where $t_{0}$ is defined by (32) and $\Lambda$ is defined by (35). We assume that the convergence rate $U_{0}$ is constant. Because little analytical progress can be made for an arbitrary power-law exponent $n$, we assume $n=3$. Despite this assumption, the solution we obtain is also valid for the Newtonian case $n=1$ if we formally set $\Lambda=0$. 
Define dimensionless (hatted) variables

$t=t_{0} \hat{t}, \quad \tau=t_{0} \hat{\tau}, \quad h=h_{0} \hat{h}, \quad Z=Z_{0} \hat{Z}$,

where $Z_{0}$ is defined by (32). Rewriting (33) and (34) in terms of the hatted variables and then immediately dropping the hats to lighten the notation, we obtain

$\frac{\partial Z}{\partial \tau}=-\frac{1}{h}$

$\dot{h}+\left[1+\Lambda(-\dot{h} / h)^{2 / 3}\right] \tau=0$

eqs (E2) and (E3) are to be solved subject to the conditions

$h(t=\tau)=1, \quad Z(\tau=t)=0$.

We now seek a solution in powers of the small parameter $\tau-t$ :

$h=1+A_{1}(\tau-t)+A_{2}(\tau-t)^{2}+\ldots$

Note that the form (E5) automatically satisfies (E4 a). Substituting (E5) into (E3) and retaining only the term of lowest order in $\tau-t$, we find that $A_{1}$ satisfies $A_{1}=\left(1+A_{1}^{2 / 3} \Lambda\right) \tau$. This cubic equation can be readily solved; expanding the solution in powers of $\Lambda$ and keeping only the lowest-order terms we obtain $A_{1}=\tau\left(1+2^{-1 / 3} \Lambda \tau^{2 / 3}\right)$, whence

$h(\tau, t)=1+\tau(\tau-t)\left(1+2^{-1 / 3} \Lambda \tau^{2 / 3}\right)$.

Next, we substitute (E6) into (E2) and expand the right-hand side in powers of $\tau-t$, retaining only the first two terms. We thereby obtain

$\frac{\partial Z}{\partial \tau}=-1+\tau(\tau-t)\left(1+2^{-1 / 3} \Lambda \tau^{2 / 3}\right)$.

Now integrate (E7) subject to (E4 b) and neglect small terms of order $t^{3}, t \tau^{2}$ and $\tau^{3}$. This yields

$Z(\tau, t)=t-\tau+\frac{3}{2^{1 / 3}} \Lambda\left(\frac{3}{88} t^{11 / 3}-\frac{1}{8} t \tau^{8 / 3}+\frac{1}{11} \tau^{11 / 3}\right)$.

Now the length of the slab is

$Z_{\max } \equiv Z(\tau=0)=t+\frac{9}{882^{1 / 3}} \Lambda t^{11 / 3}$.

Next we determine the minimum thickness $h_{\min }$ by setting to zero the derivative of (E6) with respect to $\tau$. To first order in $\Lambda$, the value of $\tau$ where $h=h_{\min }$ is

$\tau_{\min }=\frac{t}{2}+\frac{1}{12} \Lambda t^{5 / 3}$

and the minimum thickness is

$h_{\min }=1-\frac{1}{4} t^{2}-\frac{1}{8} \Lambda t^{8 / 3}$.

Inverting (E9) for $t$ as a function of $Z_{\max }$, substituting the result into (E11) and neglecting higher-order terms in $\Lambda$ and $Z_{\max }$, we obtain

$h_{\min }=1-\frac{1}{4} Z_{\max }^{2}-\frac{1}{8} \Lambda Z_{\max }^{8 / 3}$. 